DOI 10.4171/JEMS/332

Benedetta Noris · Hugo Tavares $\cdot$ Susanna Terracini · Gianmaria Verzini

\title{
Convergence of minimax structures and continuation of critical points for singularly perturbed systems
}

Received February 9, 2010

Abstract. In the recent literature, the phenomenon of phase separation for binary mixtures of Bose-Einstein condensates is understood, from the mathematical point of view, as governed by the asymptotic limit of the stationary Gross-Pitaevskii system

$$
\left\{\begin{array}{l}
-\Delta u+u^{3}+\beta u v^{2}=\lambda u \\
-\Delta v+v^{3}+\beta u^{2} v=\mu v \\
u, v \in H_{0}^{1}(\Omega), \quad u, v>0
\end{array}\right.
$$

as the interspecies scattering length $\beta$ goes to $+\infty$. For this system we consider the associated energy functionals $J_{\beta}, \beta \in(0,+\infty)$, with $L^{2}$-mass constraints, whose limit $J_{\infty}($ as $\beta \rightarrow+\infty)$ is strongly irregular. For such functionals, we construct multiple critical points via a common minimax structure, and prove convergence of critical levels and optimal sets. Moreover we study the asymptotics of the critical points.

\section{Introduction}

\subsection{Motivations}

We are interested in the nonlinear Schrödinger system

$$
\left\{\begin{array}{l}
-\Delta u+u^{3}+\beta u v^{2}=\lambda u \\
-\Delta v+v^{3}+\beta u^{2} v=\mu v, \\
u, v \in H_{0}^{1}(\Omega), \quad u, v>0,
\end{array}\right.
$$

with $\Omega$ a smooth bounded domain in $\mathbb{R}^{N}, N=2,3$, and $\lambda, \mu, \beta$ positive parameters, which arises in several physical contexts such as Bose-Einstein condensation in two hyperfine spin states (for all the physical aspects we refer, e.g., to [14, 6] and references

B. Noris, S. Terracini: Dipartimento di Matematica e Applicazioni, Università degli Studi di Milano-Bicocca, via Bicocca degli Arcimboldi 8, 20126 Milano, Italy;

e-mail: b.noris@campus.unimib.it, susanna.terracini@unimib.it

H. Tavares: University of Lisbon, CMAF, Faculty of Science, Av. Prof. Gama Pinto 2, 1649-003 Lisboa, Portugal; e-mail: htavares@ ptmat.fc.ul.pt

G. Verzini: Dipartimento di Matematica, Politecnico di Milano, p.za Leonardo da Vinci 32, 20133 Milano, Italy; e-mail: gianmaria.verzini@ polimi.it 
therein). In our case, the cubic nonlinearities $u^{3}, v^{3}$ have a positive sign, which in the literature is called the defocusing case (in opposition to the focusing one, with negative sign). For different choices of the parameters in (1), existence of solutions for this type of system has been widely studied, both for the cases of $\Omega$ bounded and for the whole $\mathbb{R}^{N}$ $([1,16,8,12]$ among others). We study solutions of (1) as nonnegative critical points of the coercive energy functional

$$
J_{\beta}(u, v)=\frac{1}{2} \int_{\Omega}\left(|\nabla u|^{2}+|\nabla v|^{2}\right) d x+\frac{1}{4} \int_{\Omega}\left(u^{4}+v^{4}\right) d x+\frac{\beta}{2} \int_{\Omega} u^{2} v^{2} d x
$$

constrained to the manifold

$$
M=\left\{(u, v) \in H_{0}^{1}(\Omega) \times H_{0}^{1}(\Omega): \int_{\Omega} u^{2} d x=\int_{\Omega} v^{2} d x=1\right\},
$$

so that $\lambda$ and $\mu$ in (1) are understood as Lagrange multipliers. This constraint represents the standard mass conservation law.

Besides the existence of ground states, because of the invariance of $J_{\beta}$ and $M$ under the $\mathbb{Z}_{2}$ action $(u, v) \mapsto(v, u)$ we expect multiple critical points of minimax type for each $\beta$. We are mainly interested in the behavior of such solutions as $\beta \rightarrow+\infty$. In the context of Bose-Einstein condensation, this models the phenomenon of phase separation (segregation) that occurs between the two different states. From the mathematical point of view, this asymptotic study of solutions has been attracting growing attention [20, 21, 19, $3,10,5,13,11,18]$. In contrast, here we analyze the asymptotics of the whole minimax structure.

While convergence of minimizers is well understood in the framework of singularly perturbed equations, this is not the case for minimax critical points. To illustrate this problem, let us consider the pointwise limit of $J_{\beta}$ as $\beta$ goes to infinity. It is the extended real valued functional defined (on $M$ ) as

$$
J_{\infty}(u, v)=\sup _{\beta>0} J_{\beta}(u, v)= \begin{cases}J_{0}(u, v) & \text { when } \int_{\Omega} u^{2} v^{2} d x=0, \\ +\infty & \text { otherwise. }\end{cases}
$$

Actually, this functional turns out to be also the $\Gamma$-limit of $J_{\beta}$ (for the definition of $\Gamma$ convergence we refer, for instance, to the book by Braides [2]). In this framework, while it is immediate to check the convergence of the minima of $J_{\beta}$ on $M$ to minima of $J_{\infty}$, it is not even obvious what should be understood as a critical point of $J_{\infty}$ (because of its strong irregularity). Also in the case when a notion of critical point is established for the limiting functional, there need not be convergence: Jerrard and Sternberg [9, Remark 4.5] exhibit an example of a family $\left\{f^{\mathcal{E}}\right\}$ of functions $\Gamma$-converging to $f$, in which a sequence of critical points of $f^{\varepsilon}$ does not converge to a critical point of $f$.

Similarly to [9], we tackle the problem from an abstract point of view (see Section 2). In fact, we consider a general family of functionals, depending on a parameter $\beta$, and its $\Gamma$-limit. These functionals share the basic property of being lower semicontinuous (with respect to a suitable topology) and nondecreasing with respect to $\beta$. After the introduction of a common minimax class, we provide a notion of critical point in connection with a 
choice of decreasing flows. The main problem is that, in our application, the limit of the gradient flows as $\beta \rightarrow+\infty$ need not itself be a continuous decreasing flow for the limiting functional (such limits were studied in [4] for the related heat equation). This prevents us from applying the recent theory of $\Gamma$-convergence of gradient flows developed in [15]. We stress that, for this reason, we do not assume any relation between the flows for $\beta<\infty$ and the limit flow.

To construct the flow for $\beta=+\infty$, we start from the observation that, in our application, the limit problem has a richer structure. Let us consider the equation

$$
-\Delta w+w^{3}=\lambda w^{+}-\mu w^{-}, \quad w \in H_{0}^{1}(\Omega),
$$

which is related to the functional $J_{\infty}$, in the case $u \cdot v \equiv 0$, as follows:

$$
J_{\infty}(u, v)=J^{*}(u-v), \quad \text { where } \quad J^{*}(w)=\frac{1}{2} \int_{\Omega}|\nabla w|^{2} d x+\frac{1}{4} \int_{\Omega} w^{4} d x
$$

(with $\int_{\Omega}\left(w^{+}\right)^{2}=\int_{\Omega}\left(w^{-}\right)^{2}=1$ ). A first suggestion for studying this relation comes from the analysis of ground state solutions: as (3) shows, if $\left(u_{\infty}, v_{\infty}\right)$ is a minimizer of $J_{\infty}$ in $M$, then $w=u_{\infty}-v_{\infty}$ is a ground state of $J^{*}$ (with the appropriate constraint). Hence, by the Lagrange multipliers theorem, $w$ solves (2). This suggests understanding the critical points of $J_{\infty}$ constrained to $M$ as pairs $(u, v)$ such that $u \cdot v \equiv 0$ and $u-v$ satisfies equation (2). As a matter of fact, we will establish, also for minimax critical points, a relation between suitable solutions $\left(u_{\beta}, v_{\beta}\right)$ of (1), for $\beta$ large, and the pairs ${ }^{1}$ $\left(w^{+}, w^{-}\right)$, where $w$ solves (2) (for suitable $\left.\lambda, \mu\right)$. Other results in the same direction have been obtained for radial functions in the recent papers $[20,19]$ for nonminimal solutions, whereas, up to our knowledge, there are no results concerning nonradial, excited states.

\subsection{A class of minimax problems}

To proceed with the exposition of our main results, we need to introduce a suitable minimax framework which is admissible for the whole family of functionals. We are inspired by a recent work by Dancer, Wei and Weth [8], where infinitely many critical levels are found, in the focusing case, by exploiting the Krasnosel'skir genus technique (see, for instance, the book by Struwe [17]) associated with the invariance of the problem when interchanging the roles of $u$ and $v$.

In carrying out our asymptotic analysis, we shall take advantage of a strong compactness property that goes beyond the usual Palais-Smale condition; to this end we are led to develop a genus theory in $L^{2}$-topology. This is the main reason why we are addressing here the defocusing case: in the focusing one, indeed, the fact that the associated Nehari manifold is not $L^{2}$-closed seems to prevent an analogous analysis. Let us consider the involution

$$
\sigma: H_{0}^{1}(\Omega) \times H_{0}^{1}(\Omega) \rightarrow H_{0}^{1}(\Omega) \times H_{0}^{1}(\Omega), \quad(u, v) \mapsto \sigma(u, v)=(v, u),
$$

\footnotetext{
1 Here, as usual, $w^{ \pm}(x)=\max \{ \pm w(x), 0\}$ denote the positive and negative parts of a function $w$.
} 
and the class of sets

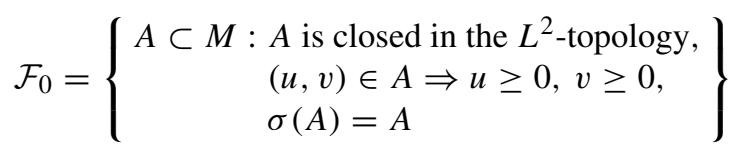

(observe that $M$ is $L^{2}$-closed and that $\sigma(M)=M$ ). We can define the Krasnosel'skiı $L^{2}$-genus in $\mathcal{F}_{0}$ in the following way.

Definition 1.1. Let $A \in \mathcal{F}_{0}$. The $L^{2}$-genus of $A$, denoted by $\gamma_{2}(A)$, is defined as

$$
\gamma_{2}(A)=\inf \left\{\begin{array}{c}
m \in \mathbb{N}: \text { there exists } f: A \rightarrow \mathbb{R}^{m} \backslash\{0\} \text { such that } \\
f \text { is continuous in the } L^{2} \text {-topology and } \\
f(\sigma(u, v))=-f(u, v) \text { for every }(u, v) \in A
\end{array}\right\} .
$$

If no $f$ as above exists, then $\gamma_{2}(A)=+\infty$, while $\gamma_{2}(\emptyset)=0$. The set of subsets with $L^{2}$-genus at least $k$ will be denoted by

$$
\mathcal{F}_{k}=\left\{A \in \mathcal{F}_{0}: \gamma_{2}(A) \geq k\right\} .
$$

Under the previous notation we define, for $0<\beta \leq+\infty$, the (candidate) critical levels

$$
c_{\beta}^{k}=\inf _{A \in \mathcal{F}_{k}} \sup _{(u, v) \in A} J_{\beta}(u, v) .
$$

In order to simplify notation, for $\beta<\infty$ we introduce a map $S_{\beta}$ such that system (1) can be rewritten as

$$
\left\{\begin{array}{l}
S_{\beta}(u, v ; \lambda, \mu)=\left(\begin{array}{l}
-\Delta u+u^{3}+\beta u v^{2}-\lambda u \\
-\Delta v+v^{3}+\beta u^{2} v-\mu v
\end{array}\right)=0 \\
u, v \in H_{0}^{1}(\Omega), \quad u, v>0
\end{array}\right.
$$

When $\beta<+\infty$, the (candidate) critical set is defined in the standard way:

$$
\mathcal{K}_{\beta}^{k}=\left\{\begin{array}{c}
(u, v) \in M: u, v \geq 0, \\
J_{\beta}(u, v)=c_{\beta}^{k}, \text { and } \\
\text { there exist } \lambda, \mu \text { such that } S_{\beta}(u, v ; \lambda, \mu)=(0,0)
\end{array}\right\} .
$$

Coming to the limiting problem, for the reasons given before, we define the critical set as

$$
\mathcal{K}_{\infty}^{k}=\left\{\begin{array}{c}
(u, v) \in M: u, v \geq 0, \\
J_{\infty}(u, v)=c_{\infty}^{k}, \text { and } \\
\text { there exist } \lambda, \mu \text { such that } u-v \text { solves (2) }
\end{array}\right\} .
$$

Our first main result states existence of critical points and of optimal sets, in the following sense.

Theorem 1.2. Let $k \in \mathbb{N}^{+}$and $0<\beta \leq+\infty$ be fixed. Then:

1. $\mathcal{K}_{\beta}^{k}$ is nonempty and compact (with respect to the $H_{0}^{1}$-topology); 
2. there exist $A_{\beta}^{k} \in \mathcal{F}_{k}$ and $\left(u_{\beta}^{k}, v_{\beta}^{k}\right) \in A_{\beta}^{k} \cap \mathcal{K}_{\beta}^{k}$ such that

$$
c_{\beta}^{k}=\max _{A_{\beta}^{k}} J_{\beta}=J_{\beta}\left(u_{\beta}^{k}, v_{\beta}^{k}\right) .
$$

As in the usual genus theory, one can also prove that if $c_{\beta}^{k}$ is the same for different $k$ 's, then the genus of $\mathcal{K}_{\beta}^{k}$ is large. This, together with suitable conditions which allow avoiding fixed points of $\sigma$ (namely $\beta$ large enough, see Lemma 3.4), provides the existence of many distinct critical points of $J_{\beta}$.

\subsection{Limits as $\beta \rightarrow+\infty$}

Since the same variational argument applies to both the $\beta$-finite and the limiting case, the next step is to compare the limiting behavior of the variational structure as $\beta \rightarrow+\infty$ with the actual behavior at $\beta=+\infty$. When $k=1$, the critical points introduced above correspond to minimal energy solutions; in this case we have

Theorem 1.3. Let $\left(u_{\beta}, v_{\beta}\right) \in M$, for $\beta \in(0,+\infty)$, be a minimizer of $J_{\beta}$ constrained to $M$. Then, up to subsequences, $\left(u_{\beta}, v_{\beta}\right)$ converges strongly in $H^{1} \cap C^{0, \alpha}$ to $\left(u_{\infty}, v_{\infty}\right)$, a minimizer of $J_{\infty}$ constrained to M. Moreover $u_{\infty}-v_{\infty}$ solves (2).

We shall obtain a proof of this result, as a byproduct of a more general one, at the end of Section 4 . For $k \geq 2$ we prove the convergence of both the critical levels and the optimal sets (in the sense of Theorem 1.2).

Theorem 1.4. Let $k \in \mathbb{N}^{+}$be fixed. As $\beta \rightarrow+\infty$ we have

1. $c_{\beta}^{k} \rightarrow c_{\infty}^{k}$;

2. if $A_{n}^{k}$ is any optimal set for $c_{\beta_{n}}^{k}$, and $\beta_{n} \rightarrow+\infty$, then the $\operatorname{set} \lim \sup _{n} A_{n}^{k}$ is optimal for $c_{\infty}^{k}$ (the limit is understood in the $L^{2}$-sense).

It is worth noticing that, in general, the convergence of the critical levels is a delicate fact (for instance, it remains an open problem in [12]). As previously mentioned, up to now there existed results in this direction that concerned only the radial case (in this case the nodal sets of the limiting equation are easier to handle). Concerning the convergence of the critical sets, we obtain the following relation.

Theorem 1.5. Let

$\mathcal{K}_{*}^{k}=\left\{\begin{array}{c}(u, v): \text { there are sequences }\left(u_{n}, v_{n}\right) \in M \text {, nonnegative, and } \beta_{n} \rightarrow+\infty \text { with } \\ \left(u_{n}, v_{n}\right) \rightarrow(u, v) \text { in } L^{2}, \\ J_{\beta_{n}}\left(u_{n}, v_{n}\right) \rightarrow c_{\infty}^{k} \text {, and } \\ S_{\beta_{n}}\left(u_{n}, v_{n}\right) \rightarrow(0,0) \text { in } L^{2}\end{array}\right\}$.

Then $\mathcal{K}_{*}^{k} \cap \mathcal{K}_{\infty}^{k}$ is not empty.

This result can be better understood in the formulation below. It makes use of the uniform Hölder bounds obtained in [13], providing that the $L^{2}$-convergences in the definitions of $\lim \sup _{\beta} \mathcal{K}_{\beta}^{k}$ and $\mathcal{K}_{*}^{k}$ are in fact strong in $H^{1} \cap C^{0, \alpha}$. 
Corollary 1.6. For every integer $k$ there exist pairs $\left(u_{\infty}, v_{\infty}\right),\left(\lambda_{\infty}, \mu_{\infty}\right)$ satisfying

$$
-\Delta\left(u_{\infty}-v_{\infty}\right)+\left(u_{\infty}-v_{\infty}\right)^{3}+\lambda_{\infty} u_{\infty}-\mu_{\infty} v_{\infty}=0
$$

at level $c_{\infty}^{k}$, and $($ sub $)$ sequences $\left(u_{\beta}, v_{\beta}\right),\left(h_{\beta}, k_{\beta}\right),\left(\lambda_{\beta}, \mu_{\beta}\right)$ satisfying

$$
\left\{\begin{array}{l}
-\Delta u_{\beta}+u_{\beta}^{3}+\beta u_{\beta} v_{\beta}^{2}-\lambda_{\beta} u_{\beta}=h_{\beta}, \\
-\Delta v_{\beta}+v_{\beta}^{3}+\beta u_{\beta}^{2} v_{\beta}-\mu_{\beta} v_{\beta}=k_{\beta} \\
u_{\beta}, v_{\beta} \in H_{0}^{1}(\Omega), \quad u, v>0
\end{array}\right.
$$

such that $\left(\lambda_{\beta}, \mu_{\beta}\right) \rightarrow\left(\lambda_{\infty}, \mu_{\infty}\right)$

$$
\left(h_{\beta}, k_{\beta}\right) \rightarrow(0,0) \text { in } L^{2} \quad \text { and } \quad\left(u_{\beta}, v_{\beta}\right) \rightarrow\left(u_{\infty}, v_{\infty}\right) \text { in } H^{1} \cap C^{0, \alpha}
$$

with $0<\alpha<1$ if $N=2$, and $0<\alpha<1 / 2$ if $N=3$.

We address the open question of finding under which conditions a solution of (2) is the limit of a sequence of solutions of (1).

The paper is structured as follows. In Section 2 we present an abstract framework of variational type; we introduce a family of functionals enjoying suitable properties and perform an asymptotic analysis. Section 3 is devoted to fitting (1) into the abstract setting; this immediately provides the convergence of the critical levels and of the optimal sets. Finally, in Section 4, we conclude the proof of the main results: we address existence and asymptotics of the critical points, leaving to Section 5 the technical details about the flows used in the deformation lemmas.

Note added in proof. The authors point out that the results contained in the recent article by E. N. Dancer, K. Wang and Z. Zhang [7] allow a substantial improvement of the statement of Corollary 1.6 of the present article. Indeed, it is proved there that all $H^{1}$-bounded families of solutions to (1) do indeed converge to a limiting profile $\left(u_{\infty}, v_{\infty}\right)$ satisfying the first equation in Corollary 1.6. This highly nontrivial fact implies that the forcing terms $\left(h_{\beta}, k_{\beta}\right)$ can be assumed to vanish identically.

Notation. In the following, $\|u\|^{2}=\int_{\Omega}|\nabla u|^{2} d x,|u|_{p}^{p}=\int_{\Omega} u^{p} d x$ (sometimes it will also denote the vectorial norm). We will refer to the topology induced on $H_{0}^{1}(\Omega) \times H_{0}^{1}(\Omega)$ by the $L^{2}(\Omega) \times L^{2}(\Omega)$ norm as the $L^{2}$-topology (and we shall denote by $\langle\cdot, \cdot\rangle_{2}$, dist $_{2}$ the associated scalar product and distance respectively). On the other hand, we will call the usual topology on $H_{0}^{1}(\Omega) \times H_{0}^{1}(\Omega)$ the $H_{0}^{1}$-topology. Finally, recall that, for a sequence $\left(A_{n}\right)_{n}$ of sets,

$$
x \in \limsup A_{n} \Leftrightarrow \text { for some } n_{k} \rightarrow+\infty \text { there exist } x_{n_{k}} \in A_{n_{k}} \text { such that } x_{n_{k}} \rightarrow x .^{2}
$$

\footnotetext{
2 This is the limit superior in the framework of the Kuratowski convergence.
} 


\section{Topological setting of a class of minimax principles}

In this section we will introduce an abstract setting of minimax type in order to obtain critical values (in a suitable sense) of a given functional. Our aim is to consider a class of functionals, each fitting in this setting, and to perform an asymptotic analysis of the variational structure. The asymptotic convergence requires some additional compactness, in the form of assumptions $(\mathcal{F} 2),\left(\mathcal{F} 2^{\prime}\right)$ below. Later on, when applying these results, this will be achieved by means of weakening the topology; the price to pay will be a loss of regularity of the functional involved. For this reason, in contrast to the usual variational schemes, our main task is to work with functionals that are only lower semicontinuous.

Let $\left(\mathcal{M}\right.$, dist) be a metric space and let us consider a set of subsets of $\mathcal{M}, \mathcal{F} \subset 2^{\mathcal{M}}$. Given a lower semicontinuous functional $J: \mathcal{M} \rightarrow \mathbb{R} \cup\{+\infty\}$, we define the minimax level

$$
c=\inf _{A \in \mathcal{F}} \sup _{x \in A} J(x),
$$

and make the following assumptions:

$(\mathcal{F} 1)$ every $A \in \mathcal{F}$ is closed in $\mathcal{M}$;

$(\mathcal{F} 2)$ there exists $c^{\prime}>c$ such that for any given $\left(A_{n}\right)_{n} \subset \mathcal{F}$ with $A_{n} \subset \mathcal{M}^{c^{\prime}}$ for every $n$, we have $\limsup _{n} A_{n} \in \mathcal{F}$, where

$$
\mathcal{M}^{c^{\prime}}=\left\{x \in \mathcal{M}: J(x) \leq c^{\prime}\right\} .
$$

Moreover from now on we will suppose that $c \in \mathbb{R}$, which in particular implies that $\mathcal{F} \neq \varnothing$ and $\emptyset \notin \mathcal{F}$. A first consequence of the compactness assumption $(\mathcal{F} 2)$ is the existence of an optimal set of the minimax procedure.

Proposition 2.1. Let $J: \mathcal{M} \rightarrow \mathbb{R} \cup\{+\infty\}$ be a lower semicontinuous functional, assume $(\mathcal{F} 2)$ and suppose moreover that $c \in \mathbb{R}$. Then there exists $\bar{A} \in \mathcal{F}$ such that $\sup _{\bar{A}} J=c$. In this situation, we will say that $\bar{A}$ is optimal for $J$ at $c$.

Proof. For every $n \in \mathbb{N}$ let $A_{n} \in \mathcal{F}$ be such that

$$
\sup _{A_{n}} J \leq c+1 / n
$$

and consider $\bar{A}:=\lim \sup _{n} A_{n}$. On one hand $\bar{A} \in \mathcal{F}$ by assumption $(\mathcal{F} 2)$, which provides $\sup _{\bar{A}} J \geq c$. On the other hand, by the definition of lim sup, for any $x \in \bar{A}$ there exists a sequence $\left(x_{n}\right)_{n}, x_{n} \in A_{n}$, such that, up to a subsequence, $x_{n} \rightarrow x$. But the lower semicontinuity implies

$$
J(x) \leq \liminf _{n} J\left(x_{n}\right) \leq \liminf _{n}\left(\sup _{A_{n}} J\right) \leq c,
$$

and the proposition follows by taking the supremum over $x \in \bar{A}$.

Due to the lack of regularity of the functional it is not obvious what should be understood as a critical set. We will give a very general definition of critical set at level $c$ by means of a "deformation", defined in some sublevel of $J$, under which the functional decreases. To this end we consider, for some $c^{\prime}>c$, a map $\eta: \mathcal{M}^{c^{\prime}} \rightarrow \mathcal{M}^{c^{\prime}}$ such that 
$(\eta 1) \eta(A) \in \mathcal{F}$ whenever $A \in \mathcal{F}, A \subset \mathcal{M}^{c^{\prime}}$;

( 22$) J(\eta(x)) \leq J(x)$ for every $x \in \mathcal{M}^{c^{\prime}}$.

We define the critical set of $J$ (relative to $\eta$ ) at level $c$ as

$$
\mathcal{K}_{c}=\{x \in \mathcal{M}: J(x)=J(\eta(x))=c\}
$$

(notice that $x \in \mathcal{M}^{c}$ and hence $\eta(x)$ is well defined). We remark that the definition depends on the choice of $\eta$. In a quite standard way, some more compactness is needed in the form of a Palais-Smale type assumption.

Definition 2.2. We say that the pair $(J, \eta)$ satisfies (PS) ${ }_{c}$ if for any given sequence $\left(x_{n}\right)_{n}$ $\subset \mathcal{M}$ such that $J\left(x_{n}\right) \rightarrow c, J\left(\eta\left(x_{n}\right)\right) \rightarrow c$, there exists $\bar{x} \in \mathcal{K}_{c}$ such that, up to a subsequence, $x_{n} \rightarrow \bar{x}$ (as above, $\eta\left(x_{n}\right)$ is well defined for $n$ sufficiently large).

Remark 2.3. Incidentally we observe that if in (PS) $)_{c}$ one would require $\bar{x}$ to be also the limit of $\eta\left(x_{n}\right)$ (we do not assume this in this section, but it will turn out to be true in the subsequent application), then $\mathcal{K}_{c}$ would coincide with the set of fixed points of $\eta$ at level $c$, providing an alternative--probably more intuitive-definition of "critical set" (relative to $\eta$ ).

As usual, $(\mathrm{PS})_{c}$ immediately implies the compactness of $\mathcal{K}_{c}$. This assumption also implies the fact that every optimal set for $J$ at level $c$ (recall Proposition 2.1) intersects $\mathcal{K}_{c}$ (which in particular is nonempty). More precisely

Theorem 2.4. Let $J: \mathcal{M} \rightarrow \mathbb{R} \cup\{+\infty\}$ be a lower semicontinuous functional, assume $(\mathcal{F} 1)$ and $(\mathcal{F} 2)$, and let $\eta: \mathcal{M}^{c^{\prime}} \rightarrow \mathcal{M}^{c^{\prime}}$ be a map such that $(\eta 1)$ and $(\eta 2)$ hold. Suppose moreover that $(J, \eta)$ satisfy (PS) $)_{c}$ and that $c \in \mathbb{R}$. Thus for every $A \in \mathcal{F}$ such that $\sup _{A} J=c$ there exists $\bar{x} \in A \cap \mathcal{K}_{c}$. In particular, $\mathcal{K}_{c}$ is nonempty.

Proof. Let $A \in \mathcal{F}$ be such that $\sup _{A} J=c$ (which exists by Proposition 2.1). By assumptions $(\eta 1)$ and $(\eta 2), \eta(A) \in \mathcal{F}$ and $\sup _{\eta(A)} J \leq c$, hence $\sup _{\eta(A)} J=c$. Thus we can find a sequence $\left(x_{n}\right)_{n} \subset A$ such that $J\left(\eta\left(x_{n}\right)\right) \rightarrow c$. By using assumption $(\eta 2)$ again, we infer

$$
c \geq J\left(x_{n}\right) \geq J\left(\eta\left(x_{n}\right)\right) \rightarrow c,
$$

and therefore (up to a subsequence) $x_{n} \rightarrow \bar{x} \in \mathcal{K}_{c}$ by (PS) ${ }_{c}$. On the other hand, since $A \in \mathcal{F}$, assumption $(\mathcal{F} 1)$ implies that $\bar{x} \in A$, which concludes the proof.

Let us now turn to the asymptotic analysis. First of all we introduce a family of functionals parameterized by $\beta \in(0,+\infty), J_{\beta}: \mathcal{M} \rightarrow \mathbb{R} \cup\{+\infty\}$, each of which is lower semicontinuous and moreover

(J) $J_{\beta_{1}}(x) \leq J_{\beta_{2}}(x)$ for every $x \in \mathcal{M}$ whenever $0<\beta_{1} \leq \beta_{2}<+\infty$.

In such a framework we define the limit functional

$$
J_{\infty}(x):=\sup _{\beta>0} J_{\beta}(x) .
$$


Lemma 2.5. For every $x_{n}, x \in \mathcal{M}$ such that $x_{n} \rightarrow x$ and $\beta_{n} \rightarrow+\infty$,

$$
J_{\infty}(x) \leq \liminf _{n} J_{\beta_{n}}\left(x_{n}\right) .
$$

In particular, $J_{\infty}$ is lower semicontinuous, and $J_{\beta} \Gamma$-converges to $J_{\infty}$.

Proof. For every fixed $\beta<+\infty$,

$$
J_{\beta}(x) \leq \liminf _{n} J_{\beta}\left(x_{n}\right) \leq \liminf _{n} J_{\beta_{n}}\left(x_{n}\right) \leq \liminf _{n} J_{\infty}\left(x_{n}\right)
$$

(we used the fact that $J_{\beta}$ is lower semicontinuous and that $J_{\beta} \leq J_{\beta_{n}}$ for $n$ sufficiently large). By taking the supremum over $\beta$ the lemma follows.

Consequently, for $0<\beta \leq+\infty$, we define the minimax levels

$$
c_{\beta}=\inf _{A \in \mathcal{F}} \sup _{x \in A} J_{\beta}(x) .
$$

Remark 2.6. Assumption (J) clearly yields

$$
\beta_{1}<\beta_{2}<+\infty \Rightarrow c_{\beta_{1}} \leq c_{\beta_{2}} \leq c_{\infty} .
$$

This suggests that any constant greater than $c_{\infty}$ is a suitable common bound for all the functionals. Hence we replace $(\mathcal{F} 2)$ with

$\left(\mathcal{F} 2^{\prime}\right)$ for any given $\left(A_{n}\right)_{n} \subset \mathcal{F}$ such that, for some $\beta, A_{n} \subset \mathcal{M}_{\beta}^{c_{\infty}+1}$ for every $n$, we have $\lim \sup _{n} A_{n} \in \mathcal{F}$,

where

$$
\mathcal{M}_{\beta}^{c^{\prime}}=\left\{x \in \mathcal{M}: J_{\beta}(x) \leq c^{\prime}\right\} .
$$

Our first main result is the convergence of both the critical levels and the optimal sets (see Proposition 2.1).

Theorem 2.7. Let $J_{\beta}: \mathcal{M} \rightarrow \mathbb{R} \cup\{+\infty\}(0<\beta<+\infty)$ be a family of lower semicontinuous functionals satisfying (J), and let $J_{\infty}$ be as before. Moreover suppose that assumption $\left(\mathcal{F} 2^{\prime}\right)$ holds, and that $c_{\beta} \in \mathbb{R}$ for every $0<\beta \leq+\infty$. Then

1. for every $0<\beta<+\infty$ there exists an optimal set for $J_{\beta}$ at $c_{\beta}$;

2. $c_{\beta} \rightarrow c_{\infty}$ as $\beta \rightarrow+\infty$;

3. if $A_{n} \in \mathcal{F}$ is optimal for $J_{\beta_{n}}$ at $c_{\beta_{n}}$ and $\beta_{n} \rightarrow+\infty$, then $A_{\infty}:=\limsup _{n} A_{n}$ is optimal for $J_{\infty}$ at $c_{\infty}$.

Proof. The first item is a direct consequence of Proposition 2.1. Now by Remark 2.6 we know that $c_{\beta}$ is monotone in $\beta$ and that $\lim c_{\beta} \leq c_{\infty}<+\infty$ by assumption. Let $\beta_{n}, A_{n}, A_{\infty}$ be as in the statement. We have $\sup _{A_{n}} J_{\beta_{1}} \leq \sup _{A_{n}} J_{\beta_{n}} \leq c_{\infty}$, therefore $A_{n} \subset\left\{J_{\beta_{1}} \leq c_{\infty}+1\right\}$ and assumption $\left(\mathcal{F} 2^{\prime}\right)$ provides $A_{\infty} \in \mathcal{F}$. Now for every $\bar{x} \in A_{\infty}$ there exists a (sub)sequence $x_{n} \rightarrow \bar{x}$ with $x_{n} \in A_{n}$. By Lemma 2.5 we have

$$
J_{\infty}(\bar{x}) \leq \liminf _{n} J_{\beta_{n}}\left(x_{n}\right) \leq \liminf _{n}\left(\sup _{A_{n}} J_{\beta_{n}}\right)=\lim _{n} c_{\beta_{n}} \leq c_{\infty} .
$$

By taking the supremum for $\bar{x} \in A_{\infty}$ (and recalling $A_{\infty} \in \mathcal{F}$ ), the theorem follows. 
Next we turn to the study of the corresponding critical sets, by introducing a family of maps $\eta_{\beta}: \mathcal{M}_{\beta}^{c_{\infty}+1} \rightarrow \mathcal{M}_{\beta}^{c_{\infty}+1}$ satisfying

$(\eta 1)_{\beta} \quad \eta_{\beta}(A) \in \mathcal{F}$ whenever $A \in \mathcal{F}, A \subset \mathcal{M}_{\beta}^{c_{\infty}+1}$;

$(\eta 2)_{\beta} \quad J_{\beta}\left(\eta_{\beta}(x)\right) \leq J_{\beta}(x)$, for every $x \in \mathcal{M}_{\beta}^{c_{\infty}+1}$.

Just as before, we define, for every $0<\beta \leq+\infty$,

$$
\mathcal{K}_{\beta}=\mathcal{K}_{c_{\beta}}=\left\{x \in \mathcal{M}: J_{\beta}(x)=J_{\beta}\left(\eta_{\beta}(x)\right)=c_{\beta}\right\} .
$$

As a straightforward consequence of Theorem 2.4, the following holds.

Theorem 2.8. Let $J_{\beta}: \mathcal{M} \rightarrow \mathbb{R} \cup\{+\infty\}(0<\beta<+\infty)$ be a family of lower semicontinuous functionals satisfying $(\mathrm{J})$, and let $J_{\infty}$ be as before. Suppose that $(\mathcal{F} 1),\left(\mathcal{F} 2^{\prime}\right)$ hold, and that, for every $0<\beta \leq+\infty, c_{\beta} \in \mathbb{R}$ and the maps $\eta_{\beta}: \mathcal{M}_{\beta}^{c_{\infty}+1} \rightarrow \mathcal{M}_{\beta}^{c_{\infty}+1}$ satisfy $(\eta 1)_{\beta}$ and $(\eta 2)_{\beta}$. Suppose moreover that the pair $\left(J_{\beta}, \eta_{\beta}\right)$ satisfies $(\mathrm{PS})_{c_{\beta}}$. Then every optimal set for $J_{\beta}$ at $c_{\beta}$ intersects $\mathcal{K}_{\beta}$, which in particular is nonempty $(\beta \leq+\infty)$.

It is now natural to wonder what is the relation between $\lim \sup \mathcal{K}_{\beta}$ and $\mathcal{K}_{\infty}$. The desired result would be their equality, which could be obtained under some suitable relations between the deformations $\eta_{\beta}$ and $\eta_{\infty}$. However, as we mentioned in the introduction, in our application such relations do not seem to hold. Instead we will assume a uniform Palais-Smale type condition, which will lead us to consider a slightly larger set than $\lim \sup \mathcal{K}_{\beta}$. Let us assume that the following holds:

(UPS) if the sequences $\left(x_{n}\right)_{n} \subset \mathcal{M}$ and $\left(\beta_{n}\right)_{n} \subset \mathbb{R}^{+}$are such that $\beta_{n} \rightarrow+\infty$ and $J_{\beta_{n}}\left(x_{n}\right) \rightarrow c_{\infty}, J_{\beta_{n}}\left(\eta_{\beta_{n}}\left(x_{n}\right)\right) \rightarrow c_{\infty}$, then there exists $\bar{x} \in \mathcal{M}$ such that, up to a subsequence, $x_{n} \rightarrow \bar{x}$ and $\eta_{\beta_{n}}\left(x_{n}\right) \rightarrow \bar{x}$

(again, since $J_{\beta_{n}}\left(x_{n}\right) \rightarrow c_{\infty}, \eta_{\beta_{n}}\left(x_{n}\right)$ is well defined for large $n$ ). It is worth pointing out explicitly the two main differences between (PS) and (UPS), apart from the dependence on $\beta$. On one hand, in the latter we do not obtain $\bar{x} \in \mathcal{K}_{\infty}$-see Remark 2.10 below. On the other hand, in (UPS) we require not only $x_{n}$ but also $\eta_{\beta_{n}}\left(x_{n}\right)$ to converge, and the limit to be the same (to enlighten this choice, see also Remark 2.3). Condition (UPS) suggests the definition of the set

$$
\mathcal{C}_{*}=\left\{\begin{array}{c}
x \in \mathcal{M}: \text { there exist sequences }\left(x_{n}\right)_{n} \subset \mathcal{M},\left(\beta_{n}\right)_{n} \subset \mathbb{R}^{+} \text {such that } \\
x_{n} \rightarrow x, \beta_{n} \rightarrow+\infty \\
J_{\beta_{n}}\left(x_{n}\right) \rightarrow c_{\infty}, \text { and } \\
J_{\beta_{n}}\left(\eta_{\beta_{n}}\left(x_{n}\right)\right) \rightarrow c_{\infty}
\end{array}\right\} .
$$

Remark 2.9. If $\left(x_{n}\right)_{n}$ is a uniform Palais-Smale sequence in the sense of (UPS), then (up to a subsequence) $x_{n} \rightarrow \bar{x} \in \mathcal{C}_{*}$.

Remark 2.10. By Theorem 2.7, it is immediate that

$$
\limsup _{\beta \rightarrow+\infty} \mathcal{K}_{\beta} \subset \mathcal{C}_{*} .
$$

Finally if $x \in \mathcal{C}_{*}$ then, by Lemma $2.5, J_{\infty}(x) \leq c_{\infty}$. Observe that the inequality may be strict (nonetheless, the following theorem will imply that for some point equality holds). 
Our final result is the following.

Theorem 2.11. Under the assumptions of Theorem 2.8, suppose moreover that (UPS) holds. Then

$$
\mathcal{C}_{*} \cap K_{\infty} \neq \emptyset
$$

More precisely, for every $\left(A_{n}\right)_{n} \subset \mathcal{F}$, with $A_{n}$ optimal for $J_{\beta_{n}}$ at $c_{\beta_{n}}$, and $\beta_{n} \rightarrow+\infty$, there exists $\bar{x} \in \mathcal{C}_{*} \cap K_{\infty} \cap \lim \sup _{n} A_{n}$.

Proof. Let $A_{n}$ be as in the statement, and take $B_{n}=\eta_{\beta_{n}}\left(A_{n}\right)$, which is also optimal for $J_{\beta_{n}}$ at $c_{\beta_{n}}$ by assumptions $(\eta 1)_{\beta_{n}},(\eta 2)_{\beta_{n}}$. Theorem 2.7 then shows that $\lim _{\sup } B_{n}=$ : $B_{\infty} \in \mathcal{F}$ is optimal for $J_{\infty}$ at $c_{\infty}$, that is, there exists

$$
\bar{y} \in B_{\infty} \cap K_{\infty} .
$$

By definition, up to a subsequence, there exists $x_{n} \in A_{n}$ such that $\eta_{\beta_{n}}\left(x_{n}\right) \rightarrow \bar{y}$. Then assumption $(\eta 2)_{\beta_{n}}$ together with Lemma 2.5 provides

$c_{\infty}=J_{\infty}(\bar{y}) \leq \liminf _{n} J_{\beta_{n}}\left(\eta_{\beta_{n}}\left(x_{n}\right)\right) \leq \liminf _{n} J_{\beta_{n}}\left(x_{n}\right) \leq \lim _{n}\left(\sup _{A_{n}} J_{\beta_{n}}\right)=\lim _{n} c_{\beta_{n}}=c_{\infty}$.

In particular this implies that $\left(x_{n}\right)_{n}$ is a Palais-Smale sequence in the sense of assumption (UPS); by using Remark 2.9 we infer that (again up to a subsequence)

$$
x_{n} \rightarrow \bar{x} \in \limsup _{n} A_{n} \cap \mathcal{C}_{*} .
$$

But (UPS) also implies $\eta_{\beta_{n}}\left(x_{n}\right) \rightarrow \bar{x}$ and hence $\bar{x}=\bar{y}$, which concludes the proof.

\section{Convergence of minimax levels}

The rest of the paper is devoted to applying (and refining) the results obtained in the previous section to the problem discussed in the introduction. In order to apply the abstract results of Section 2 we need to introduce $\mathcal{M}, \mathcal{F}$ and $\eta_{\beta}$ for the present case. In this section we deal with the asymptotics of the minimax levels and prove Theorem 1.4. The proof of the remaining results, and in particular the construction of the deformations, will be the object of the subsequent sections. Since the proof is independent of $k$, from now on we assume that $k \in \mathbb{N}^{+}$is fixed (and will often be omitted).

We define

$$
\begin{gathered}
\mathcal{M}=\left\{(u, v) \in H_{0}^{1}(\Omega) \times H_{0}^{1}(\Omega): u, v \geq 0 \text { in } \Omega,|u|_{2}=|v|_{2}=1\right\}, \\
\operatorname{dist}^{2}\left(\left(u_{1}, v_{1}\right),\left(u_{2}, v_{2}\right)\right)=\left|u_{1}-u_{2}\right|_{2}^{2}+\left|v_{1}-v_{2}\right|_{2}^{2},
\end{gathered}
$$

and

$$
J_{\beta}(u, v)=\frac{1}{2}\left(\|u\|^{2}+\|v\|^{2}\right)+\frac{1}{4} \int_{\Omega}\left(u^{4}+v^{4}\right) d x+\frac{\beta}{2} \int_{\Omega} u^{2} v^{2} d x
$$


for $0<\beta<+\infty$. Notice that the limiting functional (as introduced in Section 2) coincides with the one defined in the introduction, i.e.,

$$
J_{\infty}(u, v)=\sup _{\beta>0} J_{\beta}(u, v)= \begin{cases}J_{0}(u, v) & \text { when } \int_{\Omega} u^{2} v^{2} d x=0 \\ +\infty & \text { otherwise. }\end{cases}
$$

Moreover we set

$$
\mathcal{F}=\mathcal{F}_{k}=\left\{A \in \mathcal{F}_{0}: \gamma_{2}(A) \geq k\right\} \quad \text { (as in Definition 1.1), }
$$

which implies that the critical values $c_{\beta}$ introduced in Section 2 coincide with the values $c_{\beta}^{k}$ defined in the introduction.

Remark 3.1. We stress that for any given $c^{\prime} \in \mathbb{R}$ and $0<\beta \leq \infty$ the set

$$
\mathcal{M}_{\beta}^{c^{\prime}}=\left\{(u, v) \in \mathcal{M}: J_{\beta}(u, v) \leq c^{\prime}\right\}
$$

is $L^{2}$-compact. This is a consequence of the coercivity of the functional together with the Sobolev embedding theorem. This motivates our decision of working with this topology.

We start by presenting some properties of the $L^{2}$-genus (recall Definition 1.1 ).

Proposition 3.2. (i) Take $A \in \mathcal{F}_{0}$ and let $S^{k-1}$ be the standard $(k-1)$-sphere in $\mathbb{R}^{k}$. If there exists an $L^{2}$-homeomorphism $\psi: S^{k-1} \rightarrow$ A satisfying $\psi(-x)=\sigma(\psi(x))$ then $\gamma_{2}(A)=k$.

(ii) Consider $A \in \mathcal{F}_{k}$ and let $\eta: A \rightarrow \mathcal{M}$ be an $L^{2}$-continuous, $\sigma$-equivariant and sign-preserving map. Then $\overline{\eta(A)} \in \mathcal{F}_{k}$.

(iii) If $A \in \mathcal{F}_{0}$ is an $L^{2}$-compact set, then there exists a $\delta>0$ such that ${ }^{3} \gamma_{2}\left(\overline{N_{\delta}(A)}\right)=$ $\gamma_{2}(A)$

(iv) Let $\left(A_{n}\right)_{n \in \mathbb{N}}$ be a sequence in $\mathcal{F}_{k}$ and let $X$ be an $L^{2}$-compact subset of $\mathcal{M}$ such that $A_{n} \subset X$. Then $\lim \sup A_{n} \in \mathcal{F}_{k}$.

Proof. The proofs of the first three properties are similar to the ones of the usual genus, and therefore we omit them (see for example [17, Proposition 5.4]). As for (iv), let $A_{n}$ and $X$ be as above. By the definition of lim sup it is straightforward to check that the set $\lim \sup _{n} A_{n}$ belongs to $\mathcal{F}_{0}$, and that it is $L^{2}$-compact. We now claim that for every $\delta>0$ there exists $n_{0} \in \mathbb{N}$ such that

$$
A_{n} \subset N_{\delta}\left(\lim \sup A_{n}\right) \quad \text { for } n \geq n_{0},
$$

which together with (iii) yields the desired result. Suppose that our claim is false. Then there exist $\bar{\delta}>0, n_{k} \rightarrow+\infty$ and $\left(u_{n_{k}}, v_{n_{k}}\right) \in A_{n_{k}}$ such that $\left(u_{n_{k}}, v_{n_{k}}\right) \notin N_{\bar{\delta}}\left(\lim \sup A_{n}\right)$. But since $X$ is sequentially compact, there exists a $(u, v) \in X \subset \mathcal{M}$ such that, up to a subsequence, $\left(u_{n_{k}}, v_{n_{k}}\right) \rightarrow(u, v)$. Hence $(u, v) \in \lim \sup A_{n}$, a contradiction.

\footnotetext{
${ }^{3}$ Here $N_{\delta}(A)=\left\{(u, v) \in \mathcal{M}: \operatorname{dist}_{2}((u, v), A)<\delta\right\}$.
} 
Lemma 3.3. For every $\beta$ finite, $0 \leq c_{\beta} \leq c_{\infty}<+\infty$.

Proof. The proof relies on the fact that, given any $k \in \mathbb{N}$, we can construct a set $G_{k} \in \mathcal{F}_{0}$ with $\gamma_{2}\left(G_{k}\right)=k$. Here we use some ideas of [8, Proposition 4.3]. Indeed, consider $k$ functions $\phi_{1}, \ldots, \phi_{k} \in H_{0}^{1}(\Omega)$ such that $\phi_{i} \cdot \phi_{j}=0$ a.e. for any $i \neq j$, with $\phi_{i}^{+}, \phi_{i}^{-} \neq 0$. Define

$$
\psi: S^{k-1} \rightarrow \mathcal{M}, \quad\left(t_{1}, \ldots, t_{k}\right) \mapsto\left(\bar{t}\left(\sum_{i} t_{i} \phi_{i}\right)^{+}, \bar{s}\left(\sum_{i} t_{i} \phi_{i}\right)^{-}\right)
$$

where

$$
\bar{t}^{2}=\frac{1}{\left|\left(\sum_{i} t_{i} \phi_{i}\right)^{+}\right|_{2}^{2}}=\frac{1}{\left(\sum_{i} t_{i}^{2}\left|\phi_{i}^{+}\right|_{2}^{2}\right)}, \quad \bar{s}^{2}=\frac{1}{\left|\left(\sum_{i} t_{i} \phi_{i}\right)^{-}\right|_{2}^{2}}=\frac{1}{\left(\sum_{i} t_{i}^{2}\left|\phi_{i}^{-}\right|_{2}^{2}\right)},
$$

and $G_{k}=\psi\left(S^{k-1}\right)$. It is easy to verify that $G_{k} \in \mathcal{F}_{0}$. Since $\psi$ is an $L^{2}$-homeomorphism between $S^{k-1}$ and $G_{k}$, and $\sigma\left(\psi\left(t_{1}, \ldots, t_{k}\right)\right)=\psi\left(-t_{1}, \ldots,-t_{k}\right)$, Proposition 3.2(i) provides that $\gamma_{2}\left(G_{k}\right)=k$. Since $(u, v) \in G_{k}$ implies $u \cdot v \equiv 0$, it follows that

$$
c_{\infty} \leq \sup _{G_{k}} J_{\infty}<+\infty
$$

Finally, Remark 2.6 allows us to conclude the proof.

We are already in a position to prove the convergence of the minimax levels.

Proof of Theorem 1.4. This is a direct consequence of Theorem 2.7. Let us check its hypotheses. Under the above definitions, assumption (J) easily holds. For every $0<$ $\beta \leq+\infty, c_{\beta} \in \mathbb{R}$ (by Lemma 3.3), and moreover $\left(\mathcal{F} 2^{\prime}\right)$ holds (by recalling Remark 3.1, Proposition 3.2(iv) and by using the fact that $c_{\infty} \in \mathbb{R}$ ). Finally let us check that each $J_{\beta}$ is a lower semicontinuous functional in ( $\mathcal{M}$, dist), for $0<\beta<+\infty$. Indeed, let $\left(u_{n}, v_{n}\right),(\bar{u}, \bar{v})$ be couples of $H_{0}^{1}$ functions such that $\operatorname{dist}\left(\left(u_{n}, v_{n}\right),(\bar{u}, \bar{v})\right) \rightarrow 0$. If $\liminf _{n} J_{\beta}\left(u_{n}, v_{n}\right)=+\infty$ then there is nothing to prove; otherwise, by passing to a subsequence that achieves the lim inf, we find that $\left\|\left(u_{n}, v_{n}\right)\right\|$ is bounded. Thus, again up to a subsequence, $\left(u_{n}, v_{n}\right)$ weakly converges (in $\left.H_{0}^{1}\right)$, and, by uniqueness, the weak limit is $(\bar{u}, \bar{v})$. Then we can conclude by using the weak lower semicontinuity of $\|\cdot\|$ (and the weak continuity of the other terms in $J_{\beta}$ ).

Let us conclude this section by recalling that, if $\beta$ is sufficiently large, we can exclude the presence of fixed points of $\sigma$ in the set $\mathcal{K}_{\beta}^{k}$. As in the usual genus theory, this ensures that if two (or more) critical values coincide, then $\mathcal{K}_{\beta}^{k}$ contains an infinite number of elements.

Lemma 3.4. Let $k \in \mathbb{N}$ be fixed. There exists a (finite) number $\bar{\beta}(k)>0$, depending only on $k$, such that, for every $\bar{\beta}(k) \leq \beta \leq+\infty$,

$$
\mathcal{K}_{\beta}^{k} \cap\{(u, u) \in \mathcal{M}\}=\emptyset .
$$


Proof. When $\beta=+\infty$ the assertion holds true with no limitations on $\beta$, since $J_{\infty}(u, u)$ $<+\infty$ implies $u \equiv 0$, and $(0,0) \notin \mathcal{M}$. For $\beta<+\infty$ let us consider the problem

$$
\begin{aligned}
\inf _{(u, u) \in \mathcal{M}} J_{\beta}(u, v) & =\inf _{|u|_{2}=1}\left(\|u\|^{2}+\frac{1+\beta}{2} \int_{\Omega} u^{4} d x\right) \geq \inf _{|u|_{2}=1} \frac{1+\beta}{2|\Omega|}\left(\int_{\Omega} u^{2} d x\right)^{2} \\
& =\frac{1+\beta}{2|\Omega|} .
\end{aligned}
$$

Taking into account Lemma 3.3, the assertion of the lemma is proved once

$$
\frac{1+\beta}{2|\Omega|}>c_{k}^{\infty}
$$

But this is true if we take $\beta \geq \bar{\beta}(k)=2|\Omega| c_{k}^{\infty}$.

\section{Existence and asymptotics of the critical points}

In this section we prove the remaining results stated in the introduction. To this end we shall define suitable deformations $\eta_{\beta}$, which will allow us to apply the abstract results of Section 2 that concern the critical sets-namely Theorems 2.8 and 2.11. Afterwards, we will establish the equivalence between the critical sets defined in the introduction and the ones of Section 2.

As already mentioned, we need to choose different deformations for our purposes, for the cases $\beta<+\infty$ and $\beta=+\infty$. Let us start with the definition of $\eta_{\beta}$ for $\beta<+\infty$ (here $\beta$ is fixed). The desired map will make use of the parabolic flow associated to $J_{\beta}$ on $\mathcal{M}$. First we need to fix a relation between $(\lambda, \mu)$ and $(u, v)$.

Remark 4.1. If $(u, v) \in \mathcal{M}$ satisfies system (5) then, by testing the equations with $u$ and $v$ respectively, one immediately obtains

$$
\begin{aligned}
& \lambda=\lambda(u, v)=\frac{\int_{\Omega}\left(|\nabla u|^{2}+u^{4}+\beta u^{2} v^{2}\right) d x}{\int_{\Omega} u^{2} d x}=\int_{\Omega}\left(|\nabla u|^{2}+u^{4}+\beta u^{2} v^{2}\right) d x, \\
& \mu=\mu(u, v)=\frac{\int_{\Omega}\left(|\nabla v|^{2}+v^{4}+\beta u^{2} v^{2}\right) d x}{\int_{\Omega} v^{2} d x}=\int_{\Omega}\left(|\nabla v|^{2}+v^{4}+\beta u^{2} v^{2}\right) d x .
\end{aligned}
$$

Motivated by the previous remark and by the definition of $S_{\beta}$ (see (5)), we write, with some abuse of notation,

$$
S_{\beta}(u, v)=S_{\beta}(u, v ; \lambda(u, v), \mu(u, v)),
$$

with $\lambda, \mu$ as above. Then, for $(u, v) \in \mathcal{M}$, we consider the initial value problem with unknowns $U(x, t), V(x, t)$,

$$
\left\{\begin{array}{l}
\partial_{t}(U, V)=-S_{\beta}(U, V), \\
U(\cdot, t), V(\cdot, t) \in H_{0}^{1}(\Omega), \\
U(x, 0)=u(x), \quad V(x, 0)=v(x) .
\end{array}\right.
$$

We have the following existence result. 
Lemma 4.2. For every $(u, v) \in \mathcal{M}_{\beta}^{c_{\infty}+1}$ problem (8) has exactly one solution

$$
(U(t), V(t)) \in C^{1}\left((0,+\infty) ; L^{2}(\Omega) \times L^{2}(\Omega)\right) \cap C\left([0,+\infty) ; H_{0}^{1}(\Omega) \times H_{0}^{1}(\Omega)\right) .
$$

Moreover, for every $t>0,|(U(t), V(t))|_{2}=1$ and

$$
\frac{d}{d t} J_{\beta}(U(t), V(t))=-\left|S_{\beta}(U(t), V(t))\right|_{2}^{2} \leq 0 .
$$

We postpone to Section 5 the proofs of this result and of the subsequent properties.

Proposition 4.3. Using the notation of Lemma 4.2, the following properties hold:

(i) $U(t) \geq 0, V(t) \geq 0$, for every $(u, v) \in \mathcal{M}_{\beta}^{c_{\infty}+1}$ and $t>0$;

(ii) for every fixed $t>0$ the map $(u, v) \mapsto(U(t), V(t))$ is $L^{2}$-continuous from $\mathcal{M}_{\beta}^{c_{\infty}+1}$ into itself;

(iii) for $(u, v) \in \mathcal{M}_{\beta}^{c_{\infty}+1}$ and $s, t \in[0,+\infty)$,

$$
\operatorname{dist}((U(s), V(s)),(U(t), V(t))) \leq|t-s|^{1 / 2}\left|J_{\beta}(U(s), V(s))-J_{\beta}(U(t), V(t))\right|^{1 / 2} .
$$

All the previous results allow us to define an appropriate deformation, along with some key properties.

Proposition 4.4. Define, under the above notation,

$$
\eta_{\beta}: \mathcal{M}_{\beta}^{c_{\infty}+1} \rightarrow \mathcal{M}_{\beta}^{c_{\infty}+1}, \quad(u, v) \mapsto \eta_{\beta}(u, v)=(U(1), V(1)) .
$$

Then $\eta_{\beta}$ satisfies assumptions $(\eta 1)_{\beta}$ and $(\eta 2)_{\beta}$.

Proof. Lemma 4.2 implies that

$$
J_{\beta}\left(\eta_{\beta}(u, v)\right)=J_{\beta}(U(1), V(1)) \leq J_{\beta}(U(0), V(0))=J_{\beta}(u, v)
$$

for every $(u, v)$, which is exactly assumption $(\eta 2)_{\beta}$. This together with Proposition 4.3(i) also implies that, as stated, $\eta_{\beta}\left(\mathcal{M}_{\beta}^{c_{\infty}+1}\right) \subseteq \mathcal{M}_{\beta}^{c_{\infty}+1}$. Moreover we observe that $\eta_{\beta}$ is $\sigma$ equivariant (by the uniqueness of the initial value problem (8)) and that it is $L^{2}$-continuous (Proposition 4.3(ii)). Thus Proposition 3.2(ii) applies, yielding $\overline{\eta_{\beta}(A)} \in \mathcal{F}_{k}$. Since $A$ is $L^{2}$-compact in $\mathcal{M}$ (indeed it is a closed subset of the $L^{2}$-compact set $\mathcal{M}_{\beta}^{c_{\infty}+1}$ ), it follows that $\eta_{\beta}(A)$ is closed, and therefore assumption $(\eta 1)_{\beta}$ holds.

Before moving to the infinite case, let us prove the validity of a Palais-Smale type condition. It will be the key ingredient in showing that $\left(J_{\beta}, \eta_{\beta}\right)$ satisfies $(\mathrm{PS})_{c_{\beta}}$ according to Definition 2.2.

Lemma 4.5. Let $\left(u_{n}, v_{n}\right) \in \mathcal{M}$ be such that, as $n \rightarrow+\infty$,

$$
J_{\beta}\left(u_{n}, v_{n}\right) \rightarrow c \text { and }\left|S_{\beta}\left(u_{n}, v_{n}\right)\right|_{2} \rightarrow 0
$$

for some $c \geq 0$. Then there exists $(\bar{u}, \bar{v}) \in \mathcal{M} \cap\left(H^{2}(\Omega) \times H^{2}(\Omega)\right)$ such that, up to a subsequence,

$$
\left(u_{n}, v_{n}\right) \rightarrow(\bar{u}, \bar{v}) \text { strongly in } H_{0}^{1} \quad \text { and } \quad S_{\beta}(\bar{u}, \bar{v})=0 .
$$


Proof. Since $J_{\beta}\left(u_{n}, v_{n}\right) \rightarrow c$, we immediately infer the existence of $(\bar{u}, \bar{v}) \in \mathcal{M}$ such that $\left(u_{n}, v_{n}\right) \rightarrow(\bar{u}, \bar{v})$ weakly in $H_{0}^{1}$, up to a subsequence. Let us first prove the strong $H_{0}^{1}$-convergence. From the fact that $\left|S_{\beta}\left(u_{n}, v_{n}\right)\right|_{2} \rightarrow 0$ and that $u_{n}-\bar{u}$ is $L^{2}$-bounded, we deduce

$\left\langle S_{\beta}\left(u_{n}, v_{n}\right),\left(u_{n}-\bar{u}, 0\right)\right\rangle_{2}=\int_{\Omega}\left[\nabla u_{n} \cdot \nabla\left(u_{n}-\bar{u}\right)+\left(u_{n}^{3}+\beta u_{n} v_{n}^{2}-\lambda\left(u_{n}, v_{n}\right) u_{n}\right)\left(u_{n}-\bar{u}\right)\right] d x$ $\rightarrow 0$.

This, together with

$$
\begin{aligned}
\left|\int_{\Omega}\left(u_{n}^{3}+\beta u_{n} v_{n}^{2}-\lambda\left(u_{n}, v_{n}\right) u_{n}\right)\left(u_{n}-\bar{u}\right) d x\right| & \leq\left|u_{n}^{3}+\beta u_{n} v_{n}^{2}-\lambda\left(u_{n}, v_{n}\right) u_{n}\right|_{2}\left|u_{n}-\bar{u}\right|_{2} \\
& \leq C\left|u_{n}-\bar{u}\right|_{2} \rightarrow 0
\end{aligned}
$$

implies that $\int_{\Omega} \nabla u_{n} \cdot \nabla\left(u_{n}-\bar{u}\right) \rightarrow 0$, yielding the desired convergence. The fact that $v_{n} \rightarrow \bar{v}$ can be proved in a similar way.

Now we pass to the proof of the last part of the statement. A first observation is that

$$
\begin{aligned}
& \left|\Delta u_{n}\right|_{2}^{2}+\left|\Delta v_{n}\right|_{2}^{2} \\
& \leq 2\left|S_{\beta}\left(u_{n}, v_{n}\right)\right|_{2}^{2}+2\left|u_{n}^{3}+\beta u_{n} v_{n}^{2}-\lambda\left(u_{n}, v_{n}\right) u_{n}\right|_{2}^{2}+2\left|v_{n}^{3}+\beta u_{n}^{2} v_{n}-\mu\left(u_{n}, v_{n}\right) v_{n}\right|_{2}^{2} \leq C,
\end{aligned}
$$

which yields the weak $H^{2}$-convergence $u_{n} \rightarrow \bar{u}, v_{n} \rightarrow \bar{v}$ (up to a subsequence). As a consequence, $\left\langle S_{\beta}\left(u_{n}, v_{n}\right),(\phi, \psi)\right\rangle_{2} \rightarrow\left\langle S_{\beta}(\bar{u}, \bar{v}),(\phi, \psi)\right\rangle_{2}$ for any given $(\phi, \psi) \in L^{2}$. On the other hand, $\left|S_{\beta}\left(u_{n}, v_{n}\right)\right|_{2} \rightarrow 0$ provides that

$$
\left\langle S_{\beta}\left(u_{n}, v_{n}\right),(\phi, \psi)\right\rangle_{2} \rightarrow 0
$$

thus $S_{\beta}(\bar{u}, \bar{v})=0$ and the lemma is proved.

Let us turn to the definition of the deformation $\eta_{\infty}$. The main difficulty in this direction is that $J_{\infty}$ is finite if and only if $u v \equiv 0$, thus any flux we wish to use must preserve the disjointness of supports. As we said in the introduction, here the criticality condition will be given by equation (2). In order to overcome the lack of regularity due to the presence of the positive/negative parts in the equation, we will use a suitable gradient flow, instead of a parabolic flow. More precisely we define

$$
S_{\infty}: H_{0}^{1}(\Omega) \rightarrow H_{0}^{1}(\Omega)
$$

to be the gradient of the functional $J^{*}(w)$ (see equation (3)), constrained to the set $\int_{\Omega}\left(w^{+}\right)^{2}=\int_{\Omega}\left(w^{-}\right)^{2}=1$. If $\mathcal{L}$ denotes the inverse of $-\Delta$ with Dirichlet boundary conditions, then we will prove in Section 5 the following result. 
Lemma 4.6. Let $R_{1}, R_{2}>0$ be fixed. For every $w \in H_{0}^{1}(\Omega)$ such that

$$
\left|w^{+}\right|_{2},\left|w^{-}\right|_{2} \geq R_{1} \quad \text { and } \quad\|w\| \leq R_{2}
$$

there exist unique $\tilde{\lambda}=\tilde{\lambda}(w), \tilde{\mu}=\tilde{\mu}(w)$ such that

$$
S_{\infty}(w)=w+\mathcal{L}\left(w^{3}-\tilde{\lambda} w^{+}+\tilde{\mu} w^{-}\right) .
$$

Moreover, $\tilde{\lambda}$ and $\tilde{\mu}$ are Lipschitz continuous in $w$ with respect to the $L^{2}$-topology, with Lipschitz constants only depending on $R_{1}, R_{2}$.

For every $(u, v) \in \mathcal{M}_{\infty}^{c_{\infty}+1}$ we consider the initial value problem (with unknown $W=$ $W(t, x))$

$$
\left\{\begin{array}{l}
\partial_{t} W=-S_{\infty}(W), \\
W(\cdot, t) \in H_{0}^{1}(\Omega), \\
W(x, 0)=u(x)-v(x),
\end{array}\right.
$$

and prove existence and regularity of the solution.

Lemma 4.7. For every $(u, v) \in \mathcal{M}_{\infty}^{c_{\infty}+1}$ problem (9) has exactly one solution

$$
W(t) \in C^{1}\left((0,+\infty) ; H_{0}^{1}(\Omega)\right) \cap C\left([0,+\infty) ; H_{0}^{1}(\Omega)\right) .
$$

Moreover, for every $t,\left(W^{+}(t), W^{-}(t)\right) \in \mathcal{M}_{\infty}^{c_{\infty}+1}$ and

$$
\frac{d}{d t} J_{\infty}\left(W^{+}(t), W^{-}(t)\right)=-\left\|S_{\infty}(W(t))\right\|^{2} \leq 0 .
$$

Again, the proof of this result can be found in Section 5, together with the proof of the following properties.

Proposition 4.8. Using the notation of Lemma 4.7, the following properties hold:

(i) for every fixed $t>0$ the map $(u, v) \mapsto\left(W^{+}(t), W^{-}(t)\right)$ is $L^{2}$-continuous from $\mathcal{M}_{\infty}^{c_{\infty}+1}$ into itself;

(ii) for $(u, v) \in \mathcal{M}_{\infty}^{c_{\infty}+1}$ and $s, t \in[0,+\infty)$ we have $^{4}$

$$
\begin{aligned}
\operatorname{dist}\left(\left(W^{+}(s)\right.\right. & \left.\left., W^{-}(s)\right),\left(W^{+}(t), W^{-}(t)\right)\right) \\
& \leq C_{S}|t-s|^{1 / 2}\left|J_{\infty}\left(W^{+}(s), W^{-}(s)\right)-J_{\infty}\left(W^{+}(t), W^{-}(t)\right)\right|^{1 / 2} .
\end{aligned}
$$

Similarly to the case of $\beta$ finite, the previous properties allow one to define a suitable deformation (we omit the proof since it is similar to the case of $\beta$ finite).

Proposition 4.9. Define, under the above notation,

$$
\eta_{\infty}: \mathcal{M}_{\infty}^{c_{\infty}+1} \rightarrow \mathcal{M}_{\infty}^{c_{\infty}+1}, \quad(u, v) \mapsto \eta_{\infty}(u, v)=\left(W^{+}(1), W^{-}(1)\right) .
$$

Then $\eta_{\infty}$ satisfies assumptions $(\eta 1)_{\infty}$ and $(\eta 2)_{\infty}$.

\footnotetext{
${ }^{4}$ Here $C_{S}$ is the Sobolev constant of the embedding $H_{0}^{1} \hookrightarrow L^{2}$.
} 
Turning to the Palais-Smale condition, here is a preliminary result.

Lemma 4.10. Let $\left(u_{n}, v_{n}\right) \in \mathcal{M}_{\infty}^{c_{\infty}+1}$ be such that, as $n \rightarrow+\infty$,

$$
J_{\infty}\left(u_{n}, v_{n}\right) \rightarrow c_{\infty} \quad \text { and } \quad\left\|S_{\infty}\left(u_{n}-v_{n}\right)\right\| \rightarrow 0
$$

Then there exists $\bar{w} \in H_{0}^{1}(\Omega)$ such that, up to a subsequence,

$$
u_{n}-v_{n} \rightarrow \bar{w} \text { strongly in } H_{0}^{1} \quad \text { and } \quad S_{\infty}(\bar{w})=0 .
$$

Proof. Let $\left(w_{1}, w_{2}\right)$ be such that, up to subsequences, $u_{n} \rightarrow w_{1}$ and $v_{n} \rightarrow w_{2}$ in $H_{0}^{1}(\Omega)$. Since $J_{\infty}\left(u_{n}, v_{n}\right)<\infty$, we have $u_{n} \cdot v_{n}=0$ and therefore also $w_{1} \cdot w_{2}=0$. Denote $w_{n}=u_{n}-v_{n}$ and $\bar{w}=w_{1}-w_{2}$, so that

$$
S_{\infty}\left(u_{n}-v_{n}\right)=w_{n}+(-\Delta)^{-1}\left(w_{n}^{3}-\tilde{\lambda}\left(w_{n}\right) w_{n}^{+}+\tilde{\mu}\left(w_{n}\right) w_{n}^{-}\right) .
$$

Let us prove the $H_{0}^{1}$-convergence. First observe that $w_{n}-\bar{w}$ is bounded in $H_{0}^{1}$, which implies that $-\Delta\left(w_{n}-\bar{w}\right)$ is $H^{-1}$-bounded. Now since $\left\|S_{\infty}\left(u_{n}-v_{n}\right)\right\| \rightarrow 0$ we obtain

$$
\begin{aligned}
\left\langle-\Delta\left(w_{n}-\bar{w}\right), S_{\infty}\left(u_{n}-v_{n}\right)\right\rangle_{H^{-1}} & =\int_{\Omega}\left(\nabla w_{n} \cdot \nabla\left(w_{n}-\bar{w}\right)+w_{n}^{3}\left(w_{n}-\bar{w}\right)\right. \\
& \left.-\tilde{\lambda}\left(w_{n}\right) w_{n}^{+}\left(w_{n}-\bar{w}\right)+\tilde{\mu}\left(w_{n}\right) w_{n}^{-}\left(w_{n}-\bar{w}\right)\right) d x \rightarrow 0 .
\end{aligned}
$$

This, together with the fact that

$$
\begin{aligned}
& \mid \int_{\Omega}\left(w_{n}^{3}\left(w_{n}-\bar{w}\right)-\tilde{\lambda}\left(w_{n}\right) w_{n}^{+}\right.\left.\left(w_{n}-\bar{w}\right)+\tilde{\mu}\left(w_{n}\right) w_{n}^{-}\left(w_{n}-\bar{w}\right)\right) d x \mid \\
& \leq\left|w_{n}^{3}-\tilde{\lambda}\left(w_{n}\right) w_{n}^{+}+\tilde{\mu}\left(w_{n}\right) w_{n}^{-}\right|_{2}\left|w_{n}-\bar{w}\right|_{2} \rightarrow 0
\end{aligned}
$$

gives $\int_{\Omega} \nabla w_{n} \cdot \nabla\left(w_{n}-\bar{w}\right) \rightarrow 0$, which yields the $H_{0}^{1}$-convergence of $w_{n}$ to $\bar{w}$.

It remains to show that $S_{\infty}(\bar{w})=0$. Now, $w_{n} \rightarrow \bar{w}$ in $H_{0}^{1}$ implies that $w_{n}^{3}-$ $\tilde{\lambda}\left(w_{n}\right) w_{n}^{+}-\tilde{\mu}\left(w_{n}\right) w_{n}^{-}$is bounded in $L^{2}$, which, together with the fact that $(-\Delta)^{-1}$ is a compact operator from $L^{2}(\Omega)$ to $H_{0}^{1}(\Omega)$ provides, up to a subsequence, the convergence

$(-\Delta)^{-1}\left(w_{n}^{3}-\tilde{\lambda}\left(w_{n}\right) w_{n}^{+}-\tilde{\mu}\left(w_{n}\right) w_{n}^{-}\right) \rightarrow(-\Delta)^{-1}\left(\bar{w}^{3}-\tilde{\lambda}(\bar{w}) \bar{w}^{+}+\tilde{\mu}(\bar{w}) \bar{w}^{-}\right) \quad$ in $H_{0}^{1}(\Omega)$.

Hence also $S_{\infty}\left(u_{n}-v_{n}\right) \rightarrow S_{\infty}(\bar{w})$ in $H_{0}^{1}(\Omega)$, which concludes the proof.

We are ready to show that the deformations we have defined satisfy the remaining abstract properties required in Section 2.

Proposition 4.11. For every $0<\beta \leq+\infty$, the pair $\left(J_{\beta}, \eta_{\beta}\right)$ satisfies $(\mathrm{PS})_{c_{\beta}}$ (according to Definition 2.2). 
Proof. Let first $\beta<\infty$. Let $\left(u_{n}, v_{n}\right) \subset \mathcal{M}$ be a Palais-Smale sequence in the sense of Definition 2.2, that is, $J_{\beta}\left(u_{n}, v_{n}\right) \rightarrow c_{\beta}$ and $J_{\beta}\left(\eta_{\beta}\left(u_{n}, v_{n}\right)\right) \rightarrow c_{\beta}$. Let then $(\bar{u}, \bar{v}) \in \mathcal{M}$ be such that, up to a subsequence, $\left(u_{n}, v_{n}\right) \rightarrow(\bar{u}, \bar{v})$ in $L^{2}$. Define $\left(U_{n}(t), V_{n}(t)\right)$ as the solution of (8) with initial datum $\left(u_{n}, v_{n}\right)$ (recall that then $\left.\eta_{\beta}\left(u_{n}, v_{n}\right)=\left(U_{n}(1), V_{n}(1)\right)\right)$. By applying Proposition 4.3(iii) with $(s, t)=(0,1)$ we obtain

$$
\operatorname{dist}\left(\left(u_{n}, v_{n}\right), \eta_{\beta}\left(u_{n}, v_{n}\right)\right) \leq\left|J_{\beta}\left(u_{n}, v_{n}\right)-J_{\beta}\left(\eta_{\beta}\left(u_{n}, v_{n}\right)\right)\right|^{1 / 2} \rightarrow 0,
$$

which, together with the $L^{2}$-continuity of $\eta_{\beta}$, yields $(\bar{u}, \bar{v})=\eta_{\beta}(\bar{u}, \bar{v})$. It only remains to show that $J_{\beta}(\bar{u}, \bar{v})=c_{\beta}$. Notice that

$$
\int_{0}^{1}\left|S_{\beta}\left(U_{n}(t), V_{n}(t)\right)\right|_{2}^{2} d t=J_{\beta}\left(u_{n}, v_{n}\right)-J_{\beta}\left(\eta_{\beta}\left(u_{n}, v_{n}\right)\right) \rightarrow 0
$$

(by Lemma 4.2) and hence, for almost every $t,\left|S_{\beta}\left(U_{n}(t), V_{n}(t)\right)\right|_{2} \rightarrow 0$ (up to a subsequence). Moreover, $J_{\beta}$ being a decreasing functional under the heat flux, we have $J_{\beta}\left(U_{n}(t), V_{n}(t)\right) \rightarrow c_{\beta}$. Now Lemma 4.5 applies providing the existence of $(u, v) \in \mathcal{M}$ such that $\left(U_{n}(t), V_{n}(t)\right) \rightarrow(u, v)$ in $H_{0}^{1}$, and in particular $J_{\beta}(u, v)=c_{\beta}$. Finally the use of Proposition 4.3(iii) with $(s, t)=(0, t)$ allows us to conclude that $(u, v)=(\bar{u}, \bar{v})$, and the proof is complete.

The case $\beta=+\infty$ can be treated similarly, by replacing $(U(t), V(t))$ with $\left(W^{+}(t), W^{-}(t)\right)$ and $\left|S_{\beta}\right|_{2}$ with $\left\|S_{\infty}\right\|$.

A uniform Palais-Smale condition also holds, in the sense of assumption (UPS). The proof is very similar to the one of Proposition 4.11, and hence we omit it.

Proposition 4.12. Assumption (UPS) holds.

The properties collected in this section show that Theorems 2.8 and 2.11 apply to this framework. Thus we are in a position to conclude the proofs of the results stated in the introduction.

End of the proof of Theorem 1.2. As Theorem 2.8 holds, the last thing we have to check is that the critical set $\mathcal{K}_{\beta}$ (according to (6)) coincides with the one defined in the introduction. Again, we only present a proof in the case $\beta<+\infty$. We have to show that $J_{\beta}(u, v)=J_{\beta}(U(1), V(1))$ if and only if $S_{\beta}(u, v)=0$. But this readily follows from the fact that, for $t \in[0,1]$,

$\operatorname{dist}((u, v),(U(t), V(t)))^{2} \leq \int_{0}^{1}\left|S_{\beta}(U(\tau), V(\tau))\right|_{2}^{2} d \tau=J_{\beta}(u, v)-J_{\beta}(U(1), V(1))$,

once one observes that, by uniqueness, $(U(t), V(t)) \equiv(u, v)$ if and only if $S_{\beta}(u, v)=0$. Finally, the $H^{1}$-compactness of $\mathcal{K}_{\beta}$ comes directly from Lemmas 4.5 and 4.10.

End of the proof of Theorem 1.5. As Theorem 2.11 holds, the result is proved once we show that $\mathcal{C}_{*} \subset \mathcal{K}_{*}$. To this end, let $(u, v) \in \mathcal{C}_{*}$ and let, by definition, $\left(u_{n}, v_{n}\right) \in \mathcal{M}$ be such that $\left(u_{n}, v_{n}\right) \rightarrow(u, v)$ in $L^{2}, J_{\beta_{n}}\left(u_{n}, v_{n}\right) \rightarrow c_{\infty}$ and $J_{\beta_{n}}\left(U_{n}(1), V_{n}(1)\right) \rightarrow c_{\infty}$. By arguing exactly as in the proof of Proposition 4.11, we infer the existence of a $0 \leq t \leq 1$ 
such that $\left(U_{n}(t), V_{n}(t)\right) \rightarrow(u, v), J_{\beta_{n}}\left(U_{n}(t), V_{n}(t)\right) \rightarrow c_{\infty}$ and $\left|S_{\beta_{n}}\left(U_{n}(t), V_{n}(t)\right)\right|_{2}$ $\rightarrow 0$. Therefore $(u, v) \in \mathcal{K}_{*}$.

Proof of Corollary 1.6. The only thing left to prove is that, given any $\left(u_{n}, v_{n}\right) \in \mathcal{M}$ and $\beta_{n} \rightarrow+\infty$ such that $\left(u_{n}, v_{n}\right) \rightarrow(\bar{u}, \bar{v})$ in $L^{2}$ with $J_{\beta_{n}}\left(u_{n}, v_{n}\right) \rightarrow c_{\infty}$ and $\left|S_{\beta_{n}}\left(u_{n}, v_{n}\right)\right|_{2}$ $\rightarrow 0$, then in fact $\left(u_{n}, v_{n}\right) \rightarrow(\bar{u}, \bar{v})$ in $H^{1} \cap C^{0, \alpha}$. We shall prove that the sequence $\left(u_{n}, v_{n}\right)$ is uniformly bounded in the $L^{\infty}$-norm. This, together with the fact that, by assumption,

$$
\begin{array}{ll}
-\Delta u_{\beta}+u_{\beta}^{3}+\beta u_{\beta} v_{\beta}^{2}-\lambda_{\beta} u_{\beta}=h_{\beta} \rightarrow 0 & \text { in } L^{2} \\
-\Delta v_{\beta}+v_{\beta}^{3}+\beta u_{\beta}^{2} v_{\beta}-\mu_{\beta} v_{\beta}=k_{\beta} \rightarrow 0 & \text { in } L^{2},
\end{array}
$$

allows us to apply Theorem 1.4 of [13], which provides the desired result.

Since $J_{\beta_{n}}\left(u_{n}, v_{n}\right) \rightarrow c_{\infty}$, we infer the existence of $\lambda_{\max }, \mu_{\max } \in \mathbb{R}$ such that, up to a subsequence,

$$
\left(u_{n}, v_{n}\right) \rightarrow(\bar{u}, \bar{v}) \text { in } H_{0}^{1}, \quad \lambda\left(u_{n}, v_{n}\right) \leq \lambda_{\max }, \mu\left(u_{n}, v_{n}\right) \leq \mu_{\max }, \forall n .
$$

In order to prove uniform bounds in the $L^{\infty}$-norm, we shall apply a Brezis-Kato type argument to the sequence $\left(u_{n}, v_{n}\right)$. Suppose $u_{n} \in L^{2+2 \delta}(\Omega)$ for some $\delta>0$; we can test with $u_{n}^{1+\delta}$ the inequality

$$
-\Delta u_{n} \leq \lambda\left(u_{n}, v_{n}\right) u_{n}+h_{n}
$$

obtaining

$$
\frac{1+\delta}{(1+\delta / 2)^{2}} \int_{\Omega}\left|\nabla\left(u_{n}^{1+\delta / 2}\right)\right|^{2} d x \leq \lambda\left(u_{n}, v_{n}\right) \int_{\Omega} u_{n}^{2+\delta} d x+\int_{\Omega} h_{n} u_{n}^{1+\delta} d x .
$$

Hence, by Sobolev embedding we have ${ }^{5}$

$$
\left|u_{n}\right|_{6+3 \delta} \leq\left[C_{S}^{2} \frac{(1+\delta / 2)^{2}}{1+\delta}\right]^{\frac{1}{2+\delta}}\left[\lambda\left(u_{n}, v_{n}\right) \int_{\Omega} u_{n}^{2+\delta} d x+\int_{\Omega} h_{n} u_{n}^{1+\delta} d x\right]^{\frac{1}{2+\delta}} .
$$

Now apply the Hölder inequality to the right hand side; provided $\int_{\Omega} u_{n}^{2+2 \delta} d x \geq 1$, we have

$$
\lambda\left(u_{n}, v_{n}\right) \int_{\Omega} u_{n}^{2+\delta} d x \leq \lambda_{\max }|\Omega|^{1 / 2}\left|u_{n}\right|_{2+2 \delta}^{2+\delta} \quad \text { and } \quad \int_{\Omega} h_{n} u_{n}^{1+\delta} d x \leq\left|h_{n}\right|_{2}\left|u_{n}\right|_{2+2 \delta}^{2+\delta},
$$

hence, since $\left|h_{n}\right|_{2} \rightarrow 0$, we have proved the existence of a constant $C$, not depending on $n$ and $\delta$, such that

$$
\left|u_{n}\right|_{6+3 \delta} \leq\left[C_{S}^{2} \frac{(1+\delta / 2)^{2}}{1+\delta}\right]^{\frac{1}{2+\delta}}\left|u_{n}\right|_{2+2 \delta}
$$

Now iterate, letting

$$
\delta(1)=2, \quad 2+2 \delta(k+1)=6+3 \delta(k), \quad \text { hence } \quad \delta(k) \geq(3 / 2)^{k-1} .
$$

${ }^{5}$ Here $C_{S}$ denotes the Sobolev constant of the embedding $H_{0}^{1} \hookrightarrow L^{6}$. 
If there exist infinite values $\delta(k)$ such that $\int_{\Omega} u_{n}^{2+2 \delta(k)} d x<1$, the $L^{\infty}$-estimate is trivially proved; otherwise the previous estimates hold for $\delta(k)$ sufficiently large providing, for every $p>1$,

$$
\left|u_{n}\right|_{p} \leq C^{\prime}+\prod_{k=1}^{\infty}\left[C \frac{(1+\delta(k) / 2)^{2}}{1+\delta(k)}\right]^{\frac{1}{2+\delta(k)}}\left|u_{n}\right|_{6} .
$$

The last inequality provides the desired $L^{\infty}$-estimate since it is easy to verify that

$$
\sum_{k=1}^{\infty} \frac{1}{2+\delta(k)} \log \left[C \frac{(1+\delta(k) / 2)^{2}}{1+\delta(k)}\right]<\infty \quad \text { if } \delta(k) \geq(3 / 2)^{k-1}
$$

The same calculations clearly hold for $v_{n}$.

We conclude by giving a proof of Theorem 1.3 as a particular case of the theory we developed (although, as we mentioned, it is possible to give a more elementary proof of this result).

Proof of Theorem 1.3. The key remark in this framework is that, in fact, for every $0<$ $\beta \leq+\infty$ we can write

$$
c_{\beta}^{1}=\inf _{(u, v) \in \mathcal{M}} J_{\beta}(u, v) .
$$

More precisely,

$$
\left(u_{\beta}, v_{\beta}\right) \text { achieves } c_{\beta}^{1} \Rightarrow A_{\beta}=\left\{\left(u_{\beta}, v_{\beta}\right),\left(v_{\beta}, u_{\beta}\right)\right\} \text { is an optimal set for } J_{\beta} \text { at } c_{\beta}^{1} \text {. }
$$

Now, the $L^{2}$-convergence of the minima follows by the convergence of the optimal sets (Theorem 1.4), while the $H^{1} \cap C^{0, \alpha}$-convergence is obtained as in the previous proof.

\section{Construction of the flows}

Proof of Lemma 4.2. In order to prove local existence, we want to apply Theorem 2(b) of [22], to which we refer for further details. Let us rewrite the problem as

$$
w^{\prime}=\Delta w+F(w)
$$

where $w=(U, V), w^{\prime}=\partial_{t}(U, V), \Delta$ is understood in the vectorial sense and $F$ contains all the remaining terms. Using the notation of [22] we set $E=L^{2}(\Omega) \times L^{2}(\Omega)$ and $E_{F}=H_{0}^{1}(\Omega) \times H_{0}^{1}(\Omega)$. We find that $e^{t \Delta}$ is an analytic semigroup both on $E$ and on $E_{F}$, satisfying 6

$$
\left\|e^{t \Delta} w_{0}\right\| \leq C t^{-1 / 2}\left|w_{0}\right|_{2} \quad \text { for every } w_{0} \in E
$$

\footnotetext{
6 By using for example the expansion in eigenfunctions of $-\Delta$ in $H_{0}^{1}$, one can easily obtain the required inequality with $C=(2 e)^{-1 / 2}$.
} 
so that (2.1) in [22] holds with $a=1 / 2$. Moreover, since all the terms in $F$ are of polynomial type, it is easy to see that $F: E_{F} \rightarrow E$ is locally Lipschitz continuous, and

$$
\left|F\left(w_{0}\right)-F\left(z_{0}\right)\right|_{2} \leq \ell(r)\left\|w_{0}-z_{0}\right\|, \quad \text { with } \ell(r)=O\left(r^{p}\right) \text { as } r \rightarrow+\infty,
$$

whenever $\left\|w_{0}\right\| \leq r,\left\|z_{0}\right\| \leq r$ (for example, arguing as in Lemma 5.4, this estimate holds for $p=4)$. Now, choosing $b=1 /(2 p)<a$, it is immediate to check that

$$
\ell(r)=O\left(r^{(1-a) / b}\right)
$$

thus (2.3) in [22] is also satisfied. In order to apply Theorem 2(b), the last assumption we need to verify is that, for every $w_{0} \in H_{0}^{1}$ (which is our regularity assumption for the initial data in (8)),

$$
\underset{t \downarrow 0}{\lim \sup }\left\|t^{b} e^{t \Delta} w_{0}\right\|=0 ;
$$

but this follows on recalling that $\left\|e^{t \Delta} w_{0}\right\| \leq\left\|w_{0}\right\| .^{7}$ Therefore Theorem 2(b) and Corollary 2.1(b),(c) in [22] apply, yielding the existence of a (unique) maximal solution of (8)

$$
(U(t), V(t)) \in C^{1}\left(\left(0, T_{\max }\right) ; L^{2}(\Omega) \times L^{2}(\Omega)\right) \cap C\left(\left[0, T_{\max }\right) ; H_{0}^{1}(\Omega) \times H_{0}^{1}(\Omega)\right),
$$

with the property that if $T_{\max }<+\infty$ then $\|(U, V)\| \rightarrow+\infty$ as $t \rightarrow T_{\max }^{-}$.

Now we want to prove that $(U(t), V(t)) \in \mathcal{M}$ in its interval of definition. To this end let us consider the $C^{1}$-function

$$
\rho(t)=\int_{\Omega} U^{2}(x, t) d x,
$$

which is continuous at $t=0$. By a straightforward calculation one can see that it satisfies the initial value problem

$$
\left\{\begin{array}{l}
\rho^{\prime}(t)=a(t)(\rho(t)-1) \\
\rho(0)=1
\end{array}\right.
$$

where $a(t)=2 \lambda(U(t), V(t))$ is a continuous function. Since this problem admits only one solution, it follows that $\rho(t) \equiv 1$ in $\left[0, T_{\max }\right.$ ) (and an analogous result holds for $V(t))$. Finally, by integrating by parts (by standard regularity, $(U(t), V(t))$ belongs to $H^{2}$ for $t>0)$ and by using the fact that $\int_{\Omega} U U_{t} d x=\int_{\Omega} V V_{t} d x=0$, one can easily obtain

$$
\frac{d}{d t} J_{\beta}(U(t), V(t))=\int_{\Omega}\left(U_{t}, V_{t}\right) \cdot S_{\beta}(U, V) d x=-\left|S_{\beta}(U, V)\right|_{2}^{2} \leq 0 .
$$

This implies

$$
\|(U(t), V(t))\|^{2} \leq 2 J_{\beta}(U(t), V(t)) \leq 2 J_{\beta}(u, v)<+\infty
$$

for every $t<T_{\max }$, which provides $T_{\max }=+\infty$.

\footnotetext{
7 Again, one can obtain this inequality by expanding in eigenfunctions.
} 
Remark 5.1. Given $(u, v) \in \mathcal{M}$ let $(U, V)$ be the corresponding solution of (8). By taking into consideration inequality (10) we see that the quantities $\|(U(t), V(t))\|$, $|(U(t), V(t))|_{p}$ (with $\left.p \leq 6\right), \lambda(U(t), V(t))$ and $\mu(U(t), V(t))$ are bounded by constants which only depend on $J_{\beta}(u, v)$ (in particular, they are independent of $t$ ).

Lemma 5.2. Let $c \in C\left([0, T] ; L^{3 / 2}(\Omega)\right)$ and let $U \in C^{1}\left((0, T] ; L^{2}(\Omega)\right) \cap C([0, T]$; $\left.H_{0}^{1}(\Omega)\right)$ be a solution of

$$
\partial_{t} U-\Delta U=c(x, t) U, \quad U(\cdot, t) \in H_{0}^{1}(\Omega), U(x, 0) \geq 0 .
$$

Then $U(x, t) \geq 0$ for every $t$.

Proof. Since $c:[0, T] \rightarrow L^{3 / 2}$ we can write $|c(x, t)| \leq k+c_{1}(x, t)$, where $k$ is constant and $\left|c_{1}\right|_{3 / 2}<1 / C_{S}^{2}$ (here $C_{S}$ denotes the Sobolev constant of the embedding $H_{0}^{1}(\Omega) \hookrightarrow$ $\left.L^{6}(\Omega)\right)$. Let

$$
\rho(t)=\frac{1}{2} \int_{\Omega}\left|U^{-}(x, t)\right|^{2} d x .
$$

We see that $\rho \in C^{1}((0, T]) \cap C([0, T])$ and $\rho(0)=0$; moreover,

$$
\begin{aligned}
\rho^{\prime}(t) & =-\int_{\Omega} U^{-} \partial_{t} U d x=-\int_{\Omega}\left(U^{-} \Delta U+c(x, t)\left|U^{-}\right|^{2}\right) d x \\
& \leq-\left\|U^{-}\right\|^{2}+k\left|U^{-}\right|_{2}^{2}+\left|c_{1}\right|_{3 / 2}\left|U^{-}\right|_{6}^{2} \leq\left(-1+C_{S}^{2}\left|c_{1}\right|_{3 / 2}\right)\left\|U^{-}\right\|^{2}+k\left|U^{-}\right|_{2}^{2} \\
& \leq 2 k \rho(t) .
\end{aligned}
$$

Thus we deduce that $\rho(t) \leq e^{2 k} \rho(0)$ and the lemma follows.

Lemma 5.3. Let $w \in C^{1}\left((0,+\infty) ; L^{2}(\Omega) \times L^{2}(\Omega)\right) \cap C\left([0,+\infty) ; H_{0}^{1}(\Omega) \times H_{0}^{1}(\Omega)\right)$ be a solution of

$$
\left\{\begin{array}{l}
\partial_{t} w-\Delta w=F(w) \\
w(0)=w_{0}
\end{array}\right.
$$

where there exists a positive constant $C$ such that

$$
\int_{\Omega} F(w) \cdot w d x \leq \frac{1}{2}\|w\|^{2}+C|w|_{2}^{2} \quad \text { for every } t \geq 0 .
$$

Then there exists a constant $C(t)$ such that

$$
|w(t)|_{2} \leq C(t)\left|w_{0}\right|_{2}
$$

Proof. Let

$$
E(t)=\frac{1}{2} \int_{\Omega} w^{2}(t) d x .
$$

A straightforward computation yields

$$
E^{\prime}(t)=-\int_{\Omega}|\nabla w|^{2} d x+\int_{\Omega} F(w) \cdot w d x \leq-\frac{1}{2}\|w\|^{2}+C|w|_{2}^{2} \leq 2 C E(t),
$$

from which we obtain $E(t) \leq e^{2 C t} E(0)$, concluding the proof. 
Lemma 5.4. For $i=1,2$ take $\left(u_{i}, v_{i}\right) \in \mathcal{M}$ and let $\left(U_{i}(t), V_{i}(t)\right)$ be the corresponding solution of (8). There exists a constant $C$, only depending on $\max _{i} J_{\beta}\left(u_{i}, v_{i}\right)$, such that, for every $t$ :

1. $\left|\lambda\left(U_{1}(t), V_{1}(t)\right)-\lambda\left(U_{2}(t), V_{2}(t)\right)\right| \leq C\left(\left\|U_{1}(t)-U_{2}(t)\right\|+\left|V_{1}(t)-V_{2}(t)\right|_{2}\right)$;

2. $\left|\mu\left(U_{1}(t), V_{1}(t)\right)-\mu\left(U_{2}(t), V_{2}(t)\right)\right| \leq C\left(\left\|V_{1}(t)-V_{2}(t)\right\|+\left|U_{1}(t)-U_{2}(t)\right|_{2}\right)$.

Proof. We prove only the first inequality, since the second one is analogous. We have

$$
\begin{aligned}
\mid \lambda\left(U_{1}, V_{1}\right)- & \lambda\left(U_{2}, V_{2}\right) \mid \\
\leq & \left.\int_{\Omega}|| \nabla U_{1}\right|^{2}-\left|\nabla U_{2}\right|^{2}\left|d x+\int_{\Omega}\right| U_{1}^{4}-U_{2}^{4}\left|d x+\beta \int_{\Omega}\right| U_{1}^{2} V_{1}^{2}-U_{2}^{2} V_{2}^{2} \mid d x \\
\leq & \int_{\Omega}\left|\nabla U_{1}+\nabla U_{2}\right|\left|\nabla U_{1}-\nabla U_{2}\right| d x+\int_{\Omega}\left(U_{1}^{2}+U_{2}^{2}\right)\left|U_{1}+U_{2}\right|\left|U_{1}-U_{2}\right| d x \\
& +\beta \int_{\Omega} U_{1}^{2}\left|V_{1}+V_{2}\right|\left|V_{1}-V_{2}\right| d x+\beta \int_{\Omega} V_{2}^{2}\left|U_{1}+U_{2}\right|\left|U_{1}-U_{2}\right| d x \\
\leq & \left\|U_{1}+U_{2}\right\|\left\|U_{1}-U_{2}\right\|+\left|\left(U_{1}^{2}+U_{2}^{2}\right)\left(U_{1}+U_{2}\right)\right|_{2}\left|U_{1}-U_{2}\right|_{2} \\
& +\left|\beta U_{1}^{2}\left(V_{1}+V_{2}\right)\right|_{2}\left|V_{1}-V_{2}\right|_{2}+\left|\beta V_{2}^{2}\left(U_{1}+U_{2}\right)\right|_{2}\left|U_{1}-U_{2}\right|_{2},
\end{aligned}
$$

from which we can conclude the proof by recalling Remark 5.1 and Poincaré's inequality.

Corollary 5.5. For $i=1,2$ consider $\left(u_{i}, v_{i}\right) \in \mathcal{M}$ and let $\left(U_{i}(t), V_{i}(t)\right)$ be the corresponding solution of (8). There exists a constant $C=C(t)$, depending on $t$ (and also on $\left.\max _{i} J_{\beta}\left(u_{i}, v_{i}\right)\right)$ such that

$$
\left|\left(U_{1}(t), V_{1}(t)\right)-\left(U_{2}(t), V_{2}(t)\right)\right|_{2} \leq C(t)\left|\left(u_{1}, v_{1}\right)-\left(u_{2}, v_{2}\right)\right|_{2} .
$$

Proof. We want to apply Lemma 5.3 to $w=\left(w_{1}, w_{2}\right)=\left(U_{1}-U_{2}, V_{1}-V_{2}\right)$. Subtracting the equations for $\left(U_{1}, V_{1}\right)$ and $\left(U_{2}, V_{2}\right)$ we end up with a system like (11), thus we only need to check that

$$
F=\left(\begin{array}{l}
U_{2}^{3}-U_{1}^{3}+\beta\left(U_{2} V_{2}^{2}-U_{1} V_{1}^{2}\right)+\lambda\left(U_{1}, V_{1}\right) U_{1}-\lambda\left(U_{2}, V_{2}\right) U_{2} \\
V_{2}^{3}-V_{1}^{3}+\beta\left(U_{2}^{2} V_{2}-U_{1}^{2} V_{1}\right)+\mu\left(U_{1}, V_{1}\right) V_{1}-\mu\left(U_{2}, V_{2}\right) V_{2}
\end{array}\right)
$$

satisfies (12). To make the calculation easier, we split $F$ into four terms, after adding and subtracting some suitable quantities. The first term is

$$
F_{1}=-\left(\begin{array}{c}
\left(U_{1}^{2}+U_{1} U_{2}+U_{2}^{2}\right) w_{1} \\
\left(V_{1}^{2}+V_{1} V_{2}+V_{2}^{2}\right) w_{2}
\end{array}\right)
$$

for which we obtain, by recalling Remark 5.1,

$$
\begin{aligned}
\int_{\Omega} F_{1}(w) \cdot w d x & \leq\left|U_{1} U_{2}\right|_{3}\left|w_{1}\right|_{6}\left|w_{1}\right|_{2}+\left|V_{1} V_{2}\right|_{3}\left|w_{2}\right|_{6}\left|w_{2}\right|_{2} \\
& \leq C\left(\left\|w_{1}\right\|\left|w_{1}\right|_{2}+\left\|w_{2}\right\|\left|w_{2}\right|_{2}\right) \\
& \leq \frac{1}{2}\left(\left\|w_{1}\right\|^{2}+\left\|w_{2}\right\|^{2}\right)+C^{\prime}\left(\left|w_{1}\right|_{2}^{2}+\left|w_{2}\right|_{2}^{2}\right)
\end{aligned}
$$


(where in the last step we have used Young's inequality). The second term is

$$
F_{2}=-\beta\left(\begin{array}{c}
U_{1}\left(V_{1}+V_{2}\right) w_{2}+V_{2}^{2} w_{1} \\
V_{1}\left(U_{1}+U_{2}\right) w_{1}+U_{2}^{2} w_{2}
\end{array}\right),
$$

which immediately gives, by reasoning in the same way as above,

$$
\begin{aligned}
\int_{\Omega} F_{2}(w) \cdot w d x \leq & \beta\left(\left|U_{1}\left(V_{1}+V_{2}\right)\right|_{3}\left|w_{2}\right|_{6}\left|w_{1}\right|_{2}+\left|V_{2}^{2}\right|_{3}\left|w_{1}\right|_{6}\left|w_{1}\right|_{2}\right. \\
& \left.+\left|V_{1}\left(U_{1}+U_{2}\right)\right|_{3}\left|w_{1}\right|_{6}\left|w_{2}\right|_{2}+\left|U_{2}^{2}\right|_{3}\left|w_{2}\right|_{6}\left|w_{2}\right|_{2}\right) \\
\leq & C\left[\left\|w_{1}\right\|\left(\left|w_{1}\right|_{2}+\left|w_{2}\right|_{2}\right)+\left\|w_{2}\right\|\left(\left|w_{1}\right|_{2}+\left|w_{2}\right|_{2}\right)\right] \\
\leq & \frac{1}{2}\left(\left\|w_{1}\right\|^{2}+\left\|w_{2}\right\|^{2}\right)+C^{\prime}\left(\left|w_{1}\right|_{2}^{2}+\left|w_{2}\right|_{2}^{2}\right) .
\end{aligned}
$$

The third term is

$$
F_{3}=\left(\begin{array}{c}
\lambda\left(U_{1}, V_{1}\right) w_{1} \\
\mu\left(U_{1}, V_{1}\right) w_{2}
\end{array}\right), \quad \text { for which } \int_{\Omega} F_{3}(w) \cdot w d x \leq C|w|_{2}^{2}
$$

(where we used Remark 5.1) again. Finally, the last term is

$$
F_{4}=\left(\begin{array}{c}
\left(\lambda\left(U_{1}, V_{1}\right)-\lambda\left(U_{2}, V_{2}\right)\right) U_{2} \\
\left(\mu\left(U_{1}, V_{1}\right)-\mu\left(U_{2}, V_{2}\right)\right) V_{2}
\end{array}\right),
$$

which can be treated by using Lemma 5.4. We obtain

$$
\begin{aligned}
\int_{\Omega} F_{4}(w) \cdot w d x \leq & \left(\left\|U_{1}(t)-U_{2}(t)\right\|+\left|V_{1}(t)-V_{2}(t)\right|_{2}\right) \int_{\Omega}\left|U_{2} w_{1}\right| d x \\
& +C\left(\left\|V_{1}(t)-V_{2}(t)\right\|+\left|U_{1}(t)-U_{2}(t)\right|_{2}\right) \int_{\Omega}\left|V_{2} w_{2}\right| d x \\
\leq & \frac{1}{2}\left(\left\|w_{1}\right\|^{2}+\left\|w_{2}\right\|^{2}\right)+C^{\prime}\left(\left|w_{1}\right|_{2}^{2}+\left|w_{2}\right|_{2}^{2}\right) .
\end{aligned}
$$

Therefore $F=F_{1}+F_{2}+F_{3}+F_{4}$ satisfies (12), and hence Lemma 5.3 yields the desired result.

Proof of Proposition 4.3. Properties (i) and (ii) have been proved in Lemma 5.2 and Corollary 5.5 respectively; let us prove (iii). This is a direct consequence of the estimate on the derivative of $J_{\beta}$ given in Lemma 4.2. In fact,

$$
\begin{aligned}
\operatorname{dist}((U(s), V(s)),(U(t), V(t))) & =\left|\int_{s}^{t} \partial_{\tau}(U(\tau), V(\tau)) d \tau\right|_{2} \\
& \leq|t-s|^{1 / 2}\left(\int_{s}^{t}\left|S_{\beta}(U(\tau), V(\tau))\right|_{2}^{2} d \tau\right)^{1 / 2} \\
& =|t-s|^{1 / 2}\left|J_{\beta}(U(s), V(s))-J_{\beta}(U(t), V(t))\right|^{1 / 2}
\end{aligned}
$$

We now turn to the construction of the flux $\eta_{\infty}$. 
Proof of Lemma 4.6. By definition, $S_{\infty}$ is the projection of the gradient of $J^{*}$ at $w$ on the tangential space of the manifold $\left\{w \in H_{0}^{1}(\Omega):\left(w^{+}, w^{-}\right) \in \mathcal{M}\right\}$ at $w$, thus

$$
S_{\infty}(w)=w+\mathcal{L} w^{3}-\tilde{\lambda} \mathcal{L} w^{+}+\tilde{\mu} \mathcal{L} w^{-},
$$

where the coefficients $\tilde{\lambda}, \tilde{\mu}$ are such that $\int_{\Omega} w^{+} S_{\infty}(w) d x=\int_{\Omega} w^{-} S_{\infty}(w) d x=0$, that is,

$$
\left(\begin{array}{cc}
\int_{\Omega} w^{+} \mathcal{L} w^{+} d x & -\int_{\Omega} w^{+} \mathcal{L} w^{-} d x \\
-\int_{\Omega} w^{-} \mathcal{L} w^{+} d x & \int_{\Omega} w^{-} \mathcal{L} w^{-} d x
\end{array}\right)\left(\begin{array}{c}
\tilde{\lambda} \\
\tilde{\mu}
\end{array}\right)=\left(\begin{array}{c}
\int_{\Omega}\left(w+\mathcal{L} w^{3}\right) w^{+} d x \\
-\int_{\Omega}\left(w+\mathcal{L} w^{3}\right) w^{-} d x
\end{array}\right) .
$$

Denoting by $A$ the coefficient matrix, we compute ${ }^{8}$

$$
\operatorname{det} A=\left(\int_{\Omega}\left|\nabla \mathcal{L} w^{+}\right|^{2} d x\right)\left(\int_{\Omega}\left|\nabla \mathcal{L} w^{-}\right|^{2} d x\right)-\left(\int_{\Omega} \nabla \mathcal{L} w^{+} \cdot \nabla \mathcal{L} w^{-} d x\right)^{2} \geq 0,
$$

by the Hölder inequality, and $\operatorname{det} A=0$ if and only if $a \nabla \mathcal{L} w^{+}+b \nabla \mathcal{L} w^{-} \equiv 0$, for some $a, b$ not both zero. But this would imply that the $H_{0}^{1}(\Omega)$-function $\mathcal{L}\left(a w^{+}+b w^{-}\right)$would have an identically zero gradient and therefore $a w^{+}+b w^{-} \equiv 0$, in contradiction with the fact that, by assumption, $\left|a w^{+}+b w^{-}\right|_{2}^{2} \geq\left(a^{2}+b^{2}\right) R_{1}^{2}$. Thus the $L^{2}$-continuous function $\operatorname{det} A$ is strictly positive on the $L^{2}$-compact set $\left\{w:\left|w^{ \pm}\right|_{2} \geq R_{1},\|w\| \leq R_{2}\right\}$, i.e. it is larger than a strictly positive constant (only depending on $R_{1}, R_{2}$ ). This provides (existence, uniqueness and) an explicit expression of $\tilde{\lambda}(w)$ and $\tilde{\mu}(w)$ for any $w$ satisfying the previous assumptions. The regularity of these functions comes from such explicit expressions, once one notices that they are both products of Lipschitz continuous functions (and therefore bounded when $\|w\| \leq R_{2}$ ). Just as an example, we prove the Lipschitz continuity of the term $\int_{\Omega} w^{+} \mathcal{L} w^{3} d x$. We have ${ }^{9}$

$$
\begin{aligned}
\left|\int_{\Omega} w_{1}^{+} \mathcal{L} w_{1}^{3} d x-\int_{\Omega} w_{2}^{+} \mathcal{L} w_{2}^{3} d x\right| & \leq \int_{\Omega}\left|w_{1}^{+}-w_{2}^{+}\right| \mathcal{L} w_{1}^{3} d x+\int_{\Omega} w_{2}^{+}\left|\mathcal{L}\left(w_{1}^{3}-w_{2}^{3}\right)\right| d x \\
& \leq C\left|w_{1}^{+}-w_{2}^{+}\right|_{2}\left|w_{1}^{3}\right|_{2}+\left|w_{2}^{+}\right|_{6 / 5}\left|\mathcal{L}\left(w_{1}^{3}-w_{2}^{3}\right)\right|_{6} \\
& \leq C R_{2}^{3}\left|w_{1}-w_{2}\right|_{2}+C R_{2}\left|w_{1}^{3}-w_{2}^{3}\right|_{6 / 5} \\
& \leq C R_{2}^{3}\left|w_{1}-w_{2}\right|_{2} .
\end{aligned}
$$

All the other terms can be treated the same way.

Remark 5.6. By reasoning as at the end of the previous proof, it can be proved that, whenever $w_{1}, w_{2}$ belong to the set

$$
\left\{w \in H_{0}^{1}(\Omega):\left|w^{ \pm}\right|_{2} \geq R_{1},\|w\| \leq R_{2}\right\},
$$

there exists a constant $L$, only depending on $R_{1}, R_{2}$, such that

$$
\left|S_{\infty}\left(w_{1}\right)-S_{\infty}\left(w_{2}\right)\right|_{2} \leq L\left|w_{1}-w_{2}\right|_{2}, \quad\left\|S_{\infty}\left(w_{1}\right)-S_{\infty}\left(w_{2}\right)\right\| \leq L\left\|w_{1}-w_{2}\right\| .
$$

\footnotetext{
8 By using the identity $\int_{\Omega} f \mathcal{L} g d x=\int_{\Omega} \nabla \mathcal{L} f \cdot \nabla \mathcal{L} g d x$.

9 Remember that, by standard elliptic regularity results, both $\mathcal{L}: L^{2} \rightarrow L^{2}$ and $\mathcal{L}: L^{6 / 5} \rightarrow L^{6}$ are continuous.
} 
Proof of Lemma 4.7. Let us fix $0<R_{1}<1$ and $R_{2}^{2}>2\left(c_{\infty}+1\right)$. By Remark 5.6 we know that $-S_{\infty}$, as a map from $H_{0}^{1}(\Omega)$ into itself, is $H_{0}^{1}$-Lipschitz continuous on the set mentioned there, with Lipschitz constant only depending on $R_{1}, R_{2}$; hence we infer existence (and uniqueness) of a maximal solution of the Cauchy problem, defined on $\left[0, T_{\max }\right)$. Moreover, for any $t \in\left(0, T_{\max }\right)$, we have

$$
\frac{d}{d t}\left|W^{ \pm}(t)\right|_{2}^{2}= \pm 2 \int_{\Omega} W^{ \pm} W_{t} d x=\mp 2 \int_{\Omega} W^{ \pm} S_{\infty}(W) d x=0
$$

(by Lemma 4.6), and

$$
\begin{aligned}
\frac{d}{d t} J_{\infty}\left(W^{+}(t), W^{-}(t)\right) & =\frac{d}{d t} \int_{\Omega}\left(\frac{1}{2}|\nabla W|^{2}+\frac{1}{4} W^{4}\right) d x=\int_{\Omega}\left(-\Delta W+W^{3}\right) W_{t} d x \\
& =\int_{\Omega}-\Delta\left(W+\mathcal{L} W^{3}\right) W_{t} d x \\
& =\int_{\Omega} \nabla\left(S_{\infty}(W)+\mathcal{L}\left(\tilde{\lambda} W^{+}-\tilde{\mu} W^{-}\right)\right) \cdot \nabla\left(-S_{\infty}(W)\right) d x \\
& =-\left\|S_{\infty}(W(t))\right\|^{2} .
\end{aligned}
$$

Thus, for any $t \in\left(0, T_{\max }\right)$, we obtain $\left|W^{ \pm}(t)\right|_{2}=1>R_{1}$ and $\|W(t)\|^{2} \leq$ $2 J_{\infty}\left(W^{+}(t), W^{-}(t)\right) \leq 2 J_{\infty}(u, v)<R_{2}^{2}$. In particular this implies $T_{\max }=+\infty$, concluding the proof.

Proof of Proposition 4.8. (i) Consider $\left(u_{1}, v_{1}\right),\left(u_{2}, v_{2}\right) \in \mathcal{M}_{\infty}^{c_{\infty}+1}$ and let $W_{1}(t), W_{2}(t)$ be the corresponding solutions of (9). We notice first of all that Remark 5.6 applies, providing the existence of $L=L\left(c_{\infty}\right)$ such that

$$
\frac{d}{d t}\left|W_{1}(t)-W_{2}(t)\right|_{2}^{2} \leq 2 L\left|W_{1}(t)-W_{2}(t)\right|_{2}^{2}
$$

which implies

$$
\left|W_{1}(t)-W_{2}(t)\right|_{2}^{2} \leq e^{2 L t}\left|W_{1}(0)-W_{2}(0)\right|_{2}^{2}
$$

Therefore

$$
\begin{aligned}
\operatorname{dist}^{2}\left(\left(W_{1}^{+}(t), W_{1}^{-}(t)\right),\left(W_{2}^{+}(t), W_{2}^{-}(t)\right)\right) & \leq\left|W_{1}(t)-W_{2}(t)\right|_{2}^{2} \\
& \leq e^{2 L t}\left|W_{1}(0)-W_{2}(0)\right|_{2}^{2} \\
& \leq 2 e^{2 L t}\left(\left|u_{1}-v_{1}\right|_{2}^{2}+\left|u_{2}-v_{2}\right|_{2}^{2}\right)
\end{aligned}
$$

(ii) Notice first that

$$
\operatorname{dist}^{2}\left(\left(W^{+}(s), W^{-}(s)\right),\left(W^{+}(t), W^{-}(t)\right)\right) \leq|W(s)-W(t)|_{2}^{2} .
$$


Now, Lemma 4.7 yields

$$
\begin{aligned}
|W(s)-W(t)|_{2} & \leq C_{S}\|W(s)-W(t)\|=C_{S}\left\|\int_{S}^{t} \partial_{\tau} W(\tau) d \tau\right\| \\
& \leq C_{S}|t-s|^{1 / 2}\left(\int_{S}^{t}\left\|S_{\infty}(W(\tau))\right\|^{2} d \tau\right)^{1 / 2} \\
& =C_{S}|t-s|^{1 / 2}\left|J_{\infty}\left(W^{+}(s), W^{-}(s)\right)-J_{\infty}\left(W^{+}(t), W^{-}(t)\right)\right|^{1 / 2},
\end{aligned}
$$

and the two inequalities together conclude the proof.

Acknowledgments. This work was partially supported by MIUR, Project "Metodi Variazionali ed Equazioni Differenziali Nonlineari". The second author was supported by Fundação para a Ciência e a Tecnologia, Financiamento Base 2008 - ISFL/1/209 and FCT grant SFRH/BD/28964/ 2006.

\section{References}

[1] Ambrosetti, A., Colorado, E.: Standing waves of some coupled nonlinear Schrödinger equations. J. London Math. Soc. 75, 67-82 (2007) Zbl 1130.34014 MR 2302730

[2] Braides, A.: $\Gamma$-convergence for Beginners. Oxford Lecture Ser. Math. Appl. 22, Oxford Univ. Press, Oxford (2002) Zbl 1198.49001 MR 1968440

[3] Caffarelli, L., Lin, F.-H.: Singularly perturbed elliptic systems and multi-valued harmonic functions with free boundaries. J. Amer. Math. Soc. 21, 847-862 (2008) Zbl 1194.35138 MR 2393430

[4] Caffarelli, L., Lin, F.-H.: Nonlocal heat flows preserving the $L^{2}$ energy. Discrete Contin. Dynam. Systems 23, 49-64 (2009) Zbl 1154.35364 MR 2449068

[5] Caliari, M., Squassina, M.: Location and phase segregation of ground and excited states for 2D Gross-Pitaevskii systems. Dynam. Partial Differential Equations 5, 117-137 (2008) Zbl 1158.35322 MR 2435464

[6] Chang, S. M., Lin, C. S., Lin, T. C., Lin, W. W.: Segregated nodal domains of two-dimensional multispecies Bose-Einstein condensates. Phys. D 196, 341-361 (2004) Zbl 1098.82602 MR 2090357

[7] Dancer, E. N., Wang, K., Zhang, Z.: The limit equation for the Gross-Pitaevskii equations and S. Terracini's conjecture. J. Funct. Anal. 262, 1087-1131 (2012) Zbl pre06012164 MR 2863857

[8] Dancer, E. N., Wei, J. C., Weth, T.: A priori bounds versus multiple existence of positive solutions for a nonlinear Schrödinger system. Ann. Inst. H. Poincaré Anal. Non Linéaire 27, 953-969 (2010) Zbl 1191.35121 MR 2629888

[9] Jerrard, R. L., Sternberg, P.: Critical points via $\Gamma$-convergence: general theory and applications. J. Eur. Math. Soc. 11, 705-753 (2009) Zbl 1171.49011 MR 2538502

[10] Kuo, Y., Shieh, S.: Phase separation of multi-component Bose-Einstein condensates in optical lattices. J. Math. Anal. Appl. 347, 521-533 (2008) Zbl 1214.82009 MR 2440347

[11] Liu, Z.: Phase separation of two-component Bose-Einstein condensates. J. Math. Phys. 50, 102-104 (2009) Zbl pre05772180 MR 2573102

[12] Noris, B., Ramos, M.: Existence and bounds of positive solutions for a nonlinear Schrödinger system. Proc. Amer. Math. Soc. 138, 1681-1692 (2010) Zbl 1189.35086 MR 2587453

[13] Noris, B., Tavares, H., Terracini, S., Verzini, G.: Uniform Hölder bounds for nonlinear Schrödinger systems with strong competition. Comm. Pure Appl. Math. 63, 267-302 (2010) Zbl 1189.35314 MR 2599456 
[14] Parkins, A. S., Walls, D. F.: The physics of trapped dilute-gas Bose-Einstein condensates. Phys. Rep. 303, 1-80 (1998)

[15] Sandier, E., Serfaty, S.: Gamma-convergence of gradient flows with applications to GinzburgLandau. Comm. Pure Appl. Math. 57, 1627-1672 (2004) Zbl 1065.49011 MR 2082242

[16] Sirakov, B.: Least energy solitary waves for a system of nonlinear Schrödinger equations in $\mathbb{R}^{n}$. Comm. Math. Phys. 271, 199-221 (2007) Zbl 1147.35098 MR 2283958

[17] Struwe, M.: Variational Methods. Springer, Berlin (1990) Zbl 0746.49010 MR 1078018

[18] Tavares, H., Terracini, S.: Regularity of the nodal set of segregated critical configurations. Preprint (2010)

[19] Terracini, S., Verzini, G.: Multipulse phases in $k$-mixtures of Bose-Einstein condensates. Arch. Ration. Mech. Anal. 194, 717-741 (2009) Zbl 1181.35069 MR 2563622

[20] Wei, J., Weth, T.: Radial solutions and phase separation in a system of two coupled Schrödinger equations. Arch. Ration. Mech. Anal. 190, 83-106 (2008) Zbl 1161.35051 MR 2434901

[21] Wei, J., Weth, T.: Asymptotic behaviour of solutions of planar elliptic systems with strong competition. Nonlinearity 21, 305-317 (2008) Zbl 1132.35482 MR 2384550

[22] Weissler, F. B.: Local existence and nonexistence for semilinear parabolic equations in $L^{p}$. Indiana Univ. Math. J. 29, 79-102 (1980) Zbl 0443.35034 MR 0554819 